\title{
Well-posed and Discretely Stable Perfectly Matched Layers for Elastic Wave Equations in Second Order Formulation
}

\author{
Kenneth Duru ${ }^{1, *}$, Gunilla Kreiss ${ }^{1}$ \\ ${ }^{1}$ Division of Scientific Computing, Department of Information Technology, Uppsala \\ University Sweden.
}

\begin{abstract}
We present a well-posed and discretely stable perfectly matched layer for the anisotropic (and isotropic) elastic wave equations without first re-writing the governing equations as a first order system. The new model is derived by the complex coordinate stretching technique. Using standard perturbation methods we show that complex frequency shift together with a chosen real scaling factor ensures the decay of eigen-modes for all relevant frequencies. To buttress the stability properties and the robustness of the proposed model, numerical experiments are presented for anisotropic elastic wave equations. The model is approximated with a stable node-centered finite difference scheme that is second order accurate both in time and space.
\end{abstract}

AMS subject classifications: 35B35, 35L05, 35L15, 37C75.

Key words: Perfectly matched layer, Well-posedness, Stability, Hyperbolicity, Elastic waves.

\section{Introduction}

Perfectly matched layers (PML) have since the introduction [3], emerged as a standard non-reflecing boundary closure for many wave propagation problems. In this paper we consider linear, anisotropic elasto-dynamics in two space dimensions. Equations describing the dynamics are usually derived via Newtons law connecting accelaration and force, thus yielding a second order system (in both time and space) for the displacements. The system is hyperbolic, and by introducing suitable variables the model can be rewritten as a hyperbolic first order system. PMLs for elasto-dynamics are usully derived from the first order formulation $[4,5,23]$. This is also the case for other hyperbolic systems that naturally come in second order formulation, like the standard wave equation.

However, there are several advantages with using the second order formulation. The first order formulation requires more variables, and it introduces a new wave with zero

*Corresponding author. Email addresses: kenneth.duru@it.uu.se (K. Duru), gunilla.kreiss@it.uu.se (G. Kreiss) 
wave speed. Also, in many cases a straightforward discretization of the first order formulation introduces high frequency spurious modes. In this paper we construct a PML for the second order equations of linear, anisotropic elasto-dynamics in two space dimensions without first rewriting the equations as a first order system. By construction the PML is perfectly matched, but there is no guarantee that all solutions decay with time. The analysis of temporal stability is therefore a main topic of research. In [4], the geometric stability condition was formulated, and found to be a necessary condition for stability of the split field PML. In [5], it was proved to be necessary also for stability of a modal PML, even though the complex frequency shift had a stablizing effect.

The aim of this paper is to construct efficient layers based on the second order equations, for all materials, and also those violating the geometric stability condition. The PML equations are derived using a complex coordinate stretching technique, $[6,17]$. We include a grid stretching parameter and a complex frequancy shift. One advantage of this approach is that we can choose auxilary variables so that the resulting system is strongly hyperbolic.

In computations using standard second order central finite differences, our PML behaves dramatically better than the corresponding first order PMLs, for materials where the geometric stability condition is violated. In many cases no growth is seen in the computation even at very late times. To understand this behaviour we start by applying a standard perturbation analysis to our PML at constant coefficients. The result is that our PML suffers from the same high frequency instability as the above mentioned first order PMLs for the geometric stability violating materials. A large part of the paper is dedicted to understanding why our PML behaves in this stable way, and how the stable behaviour can be enhanced.

From the analysis of the continous constant coefficient problem we know that the instability appears only at sufficiently high spatial frequencies. If these frequencies are not well resolved, the the discrete behaviour may be completely different. A straight forward computation of the temporal eigenvalues corresponding to the discrete spatial operator in a constant coefficient setting shows that if unstable modes are not well resolved, they are in fact stable in the discrete setting. We have investigated how the grid stretching parameter can be used to enhance this effect.

A second reason is the stablizing effect of corner regions. When the layers are used as boundary closures completely surrounding a domain there are usually corner regions. We use the same perturbation technique as above applied to a constant coefficient corner problem, and find that our PML is stable in the corner region. In computations we have observed that the bulk of an unstable mode typically is localized to part of layer and propagating tangentially while the amplitude grows. Eventually the bulk of the unstable mode moves into a corner region and is damped.

The paper is organized as follows. In Section 2 we introduce the elastic wave equation, the materials treated in the paper, and their corresponding dispersion relations. A brief review of existing layers and there properties are given, followed by a derivation of our layer. The basic mathematical properties such as perfect matching, hyperbolicity 
and wellposedness are discussed. Section 3 is devoted to stability analysis. In section 4 we present numerical calculations both as illustration of our theoretical results, and to further explore the properties of our layer. We close section 4 by investigating the efficiency of the layer. We present computations where we show that it is possible to chose parameters related to the spatial step size yielding reflections from the layer that decay at the same rate as the numerical discretization error $\left(\sim h^{2}\right)$. In section 5 a brief conclusion is offered.

\section{Elastic waves and damping Layers}

In this section, the second order equations of linear elastodynamics (in orthotropic media) in two space dimensions will be stated and we will derive the PML model using complex coordinate stretching technique.

\subsection{Elastic Waves}

Using Einstein's convention of summation and neglecting body forces, the equation of continuum can be written

$$
\rho \frac{\partial^{2} u_{i}}{\partial t^{2}}=\frac{\partial \sigma_{i, j}}{\partial x_{j}}, \quad i, j=1,2
$$

Here $\rho$ is the density and $u_{1}, u_{2}$ are the displacement, $\sigma_{i, j}$ is the stress tensor, which is related to the tensor of deformation

$$
\epsilon_{i, j}(u)=\frac{1}{2}\left(\frac{\partial u_{i}}{\partial x_{j}}+\frac{\partial u_{j}}{\partial x_{i}}\right) .
$$

By Hook's law

$$
\sigma_{i, j}=C_{i j k l} \epsilon_{k l} .
$$

The fourth-order tensor $\mathrm{C}$ of elastic coefficients satisfies the classical symmetries

$$
C_{i j k l}=C_{j i k l}=C_{k l i j}
$$

It is also positive definite,

$$
C_{i j k l} \psi_{i j} \psi_{k l} \geq\|\psi\|^{2}=\alpha \psi_{i j} \psi_{i j}
$$

for all symmetric tensor $\psi_{i j}$, see [4]. The symmetry of $C$ allows us to simplify the equations by using the scheme

$$
C_{i j k l}=c_{p(i, j), p(k, l)}
$$

where $p(i, j)=i$, if $i=j, \quad p(i, j)=i+j$, if $i \neq j$.

In an Orthotropic medium whose principal axes coincides with the $(x, y)$ axes, we have $c_{13}=c_{23}=0$, hence 


$$
C=\left(\begin{array}{ccc}
c_{11} & c_{12} & 0 \\
c_{21} & c_{22} & 0 \\
0 & 0 & c_{33}
\end{array}\right) .
$$

Now equation (2.1) can be formally written as

$$
\mathbf{u}_{t t}=A_{1} \mathbf{u}_{x x}+A_{2} \mathbf{u}_{y y}+A_{3} \mathbf{u}_{x y}
$$

where $\mathbf{u}=\left[u_{1}, u_{2}\right]^{T}, \quad A_{1}=\left(\begin{array}{ll}a & 0 \\ 0 & c\end{array}\right), \quad A_{2}=\left(\begin{array}{ll}c & 0 \\ 0 & b\end{array}\right), \quad A_{3}=\left(\begin{array}{cc}0 & d \\ d & 0\end{array}\right)$.

Here $a=c_{11}, b=c_{22}, c=c_{33}, d=c_{33}+c_{21}$. For convenience we have also assumed $\rho=1$. It is easy to show that (2.3) is strongly hyperpolic and strongly well-posed.

\subsection{Plane waves and slowness diagrams}

In order to understand the wave propagation properties of the model (2.3) it is useful to consider wave-like solutions

$$
\mathbf{u}=\mathbf{u}_{0} e^{s t-i k_{x} x-i k_{y} y}, \quad \mathbf{u}_{0} \in \mathbb{R}^{n}, \quad k_{x}, k_{y}, x, y, z \in \mathbb{R}, \quad 0 \leq t .
$$

In equation (2.4), $\left(k_{x}, k_{y}\right) \in \mathbb{R}^{2}$ is the wave vector, and $\mathbf{u}_{0}$ is a vector of constant amplitude called the polarization vector. By inserting (2.4) in (2.3) we have a solvability condition (2.5), often called the dispersion relation

$$
\begin{aligned}
& F_{0}\left(s, k_{x}, k_{y}\right) \equiv \operatorname{det}\left(s^{2} I+\Psi\left(k_{x}, k_{y}\right)\right)=0, \\
& \Psi\left(k_{x}, k_{y}\right)=A_{1} k_{x}^{2}+A_{2} k_{y}^{2}+A_{3} k_{x} k_{y} .
\end{aligned}
$$

The polarization vector $\mathbf{u}_{0}$ is the eigenvector of $\Psi\left(k_{x}, k_{y}\right)$, with associated egeinvalue $-s^{2}$. By evaluating (2.5) for (2.3) we have

$$
\begin{aligned}
F_{0}\left(s, k_{x}, k_{y}\right) & \equiv s^{4}+\left((a+c) k_{x}^{2}+(b+c) k_{y}^{2}\right) s^{2}+a c k_{x}^{4} \\
& +b c k_{y}^{4}+\left(a b+c^{2}-d^{2}\right) k_{x}^{2} k_{y}^{2}=0 .
\end{aligned}
$$

It should be noted that the polynomial (2.6) has four purely imaginary roots, corresponding to the quasi-P and quasi-S waves. We will refer to these modes as the physical modes.

If we write $s=i \omega\left(k_{x}, k_{y}\right)$, where $\omega \in \mathbb{R}$ is called the temporal frequency, we can also introduce

$$
\begin{aligned}
& K=\left(\frac{k_{x}}{|k|}, \frac{k_{y}}{|k|}\right), \text { the normalized propagation direction, } \\
& V_{p}=\left(\frac{\omega}{k_{x}}, \frac{\omega}{k_{y}}\right), \text { the phase velocity, } \\
& S=\left(\frac{k_{x}}{\omega}, \frac{k_{y}}{\omega}\right), \text { the slowness vector, } \\
& V_{g}=\nabla_{k} \omega\left(k_{x}, k_{y}\right), \quad \text { the group velocity, } \\
& \text { and } \quad|k|=\sqrt{k_{x}^{2}+k_{y}^{2}} .
\end{aligned}
$$


By inspection (2.6) is homogeneous in $\omega, k_{x}, k_{y}$, we can re-write (2.6) as

$$
F_{0}(i, S)=0
$$

The wave propagation properties of a certain medium can be described by plotting the slowness diagrams defined by points in the $S$-plane satisfying (2.8). If we further assume $c_{11} \neq c_{33}$ and $c_{22} \neq c_{33}$, the group velocity can be expressed as

$$
V_{g}=\nabla_{k} \omega\left(k_{x}, k_{y}\right)=-\left(\frac{\partial F_{0}\left(i \omega, k_{x}, k_{y}\right)}{\partial \omega}\right)^{-1} \nabla_{k} F_{0}\left(i \omega, k_{x}, k_{y}\right)
$$

That is the group velocity is normal to the slowness curve.

In [4] Theorem 2, a necessary temporal stability condition for the split-field PML is established see Figure 1. The condition states:

Definition 1. Geometric stability condition

1. The geometric stability condition in the $x$-direction (parallel to the vertical axis) is $S_{x} \times\left(V_{g}\right)_{x} \geq 0$, for all points on the slowness curve.

2. The geometric stability condition in the $y$-direction (parallel to the horizontal axis) is

$S_{y} \times\left(V_{g}\right)_{y} \geq 0$, for all points on the slowness curve.

Here $V_{g}=\left(\left(V_{g}\right)_{x},\left(V_{g}\right)_{y}\right)$ and $S=\left(S_{x}, S_{y}\right)$.

The material parameters we shall consider in this paper are presented in Table 1 . The anisotropic Material MA1 with the slowness diagram Figure 2(a) severely violates the geometric stability conditions in both $x$ - and $y$-directions, it is also the same as Material III in [5]. The anisotropic material MA2 (Apatite) marginally violates the geometric stability condition in both $x$ - and $y$-direction, see Figure 2(b) and has been studied in [16]. The material MA3 violates the geometric stability condition only in the $y$-direction, see Figure 2(c) while the anisotropic material MA4 (Zinc crystal) violates the stability condition in the $x$-direction, see also see Figure 2(d) and has also been studied in [16]. For the Materials MA1 and MA2, the classical PML in both $x$ - and $y$-directions will be unstable, while the $y$-dependent PML for material MA3 will be unstable and the $x$-dependent PML for material MA4 will be unstable. The anisotropic Material MB is the same as Material I in [5] and does not violate the stability condition, the classical PML for the Material MB will be expected to be stable. Though Material MC (same as Material IV in [5]) does not not violate the geometric stability condition, it has a generic weak instability as demonstrated in [4]. 


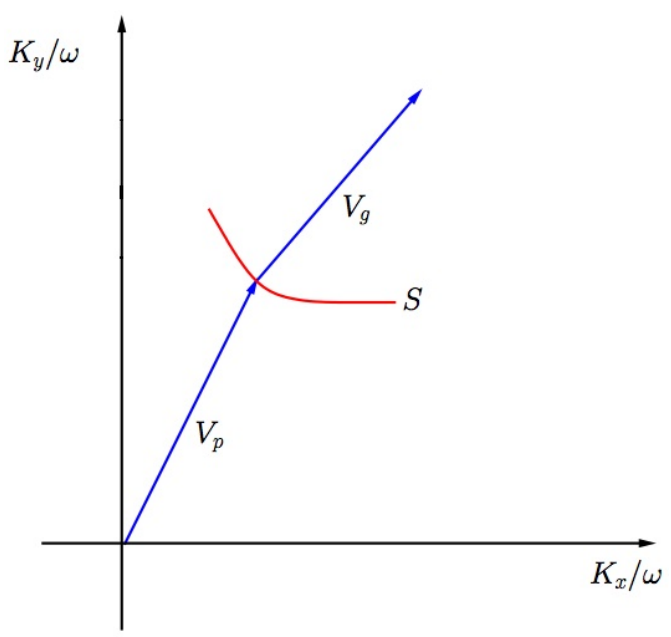

(a) Stable

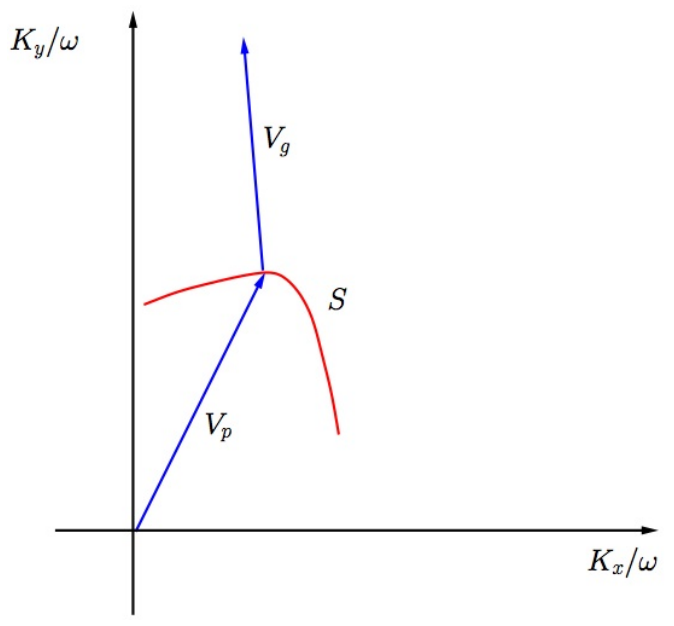

(b) Unstable

Figure 1: Geometric stability condition.

\begin{tabular}{|l||l|l||l|l|}
\hline \multirow{2}{*}{ Material } & \multicolumn{4}{|c|}{ Elastic Coefficients } \\
\cline { 2 - 5 } & $c_{11}$ & $c_{22}$ & $c_{33}$ & $c_{12}$ \\
\hline \hline MA1 & 4.00 & 20.00 & 2.00 & 7.50 \\
\hline MA2 & 16.70 & 14.00 & 6.63 & 6.60 \\
\hline MA3 & 4.00 & 20.00 & 2.00 & 4.90 \\
\hline MA4 & 16.50 & 6.20 & 3.96 & 5.00 \\
\hline MB & 4.00 & 20.00 & 2.00 & 3.80 \\
\hline MC & 10.00 & 20.00 & 6.00 & 2.50 \\
\hline
\end{tabular}

Table 1: Elastic coefficients of the orthotropic materials

\subsection{Damping layers for elastic wave equations}

Several damping layers exist for the elastic wave equations, [4, 5, 8, 23]. Many of these layers are only weakly hyperbolic and weakly well-posed. In many cases the existing layers also support exponentially growing solution. This shortcoming, for the classical PML for elastic waves as reported in [4] was in part corrected in a paper by Appelö \& Kreiss [5] by adding an additional parameter (the complex frequency shift) in a modal PML in first order formulation. For materials that violate the geometric stability condition, the construction of a stable and efficient absorbing layer has remained a challenge.

In a very recent work [16], Meza-Fajardo and Papageogiou proposed a new model, the multi-axial perfectly matched layer (M-PML). Their model is based on a damping profile that varies in more than one direction. This approach may give a stable model for some materials but it generates non-trivial reflections from the interface of the layer and 


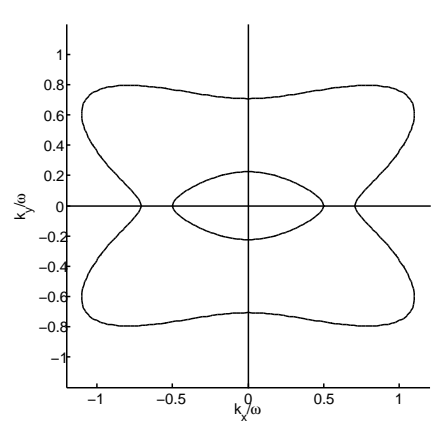

(a) MA1.

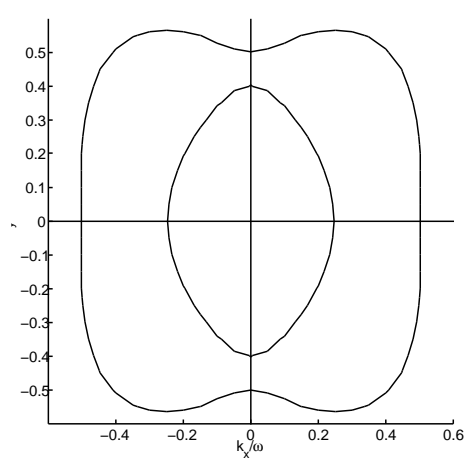

(d) MA4.

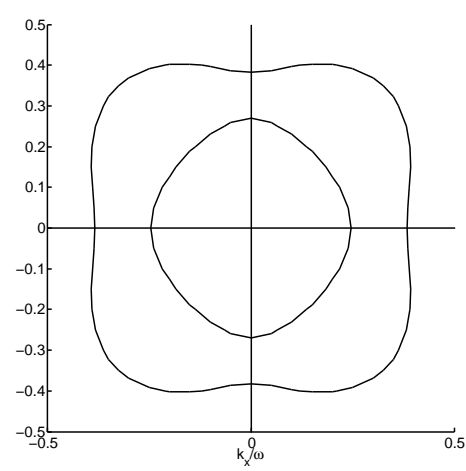

(b) MA2.

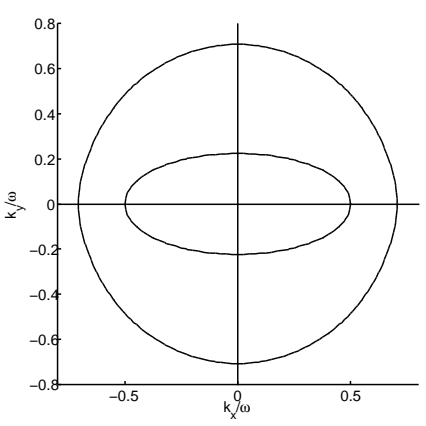

(e) MB.

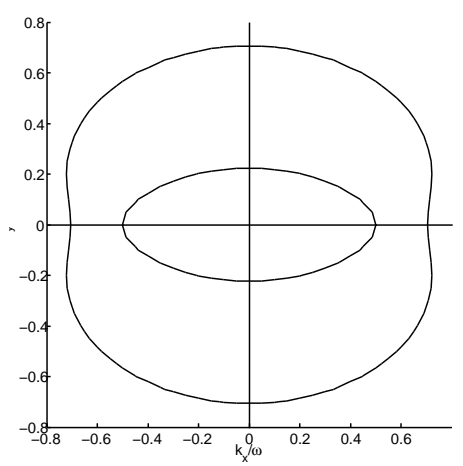

(c) MA3.

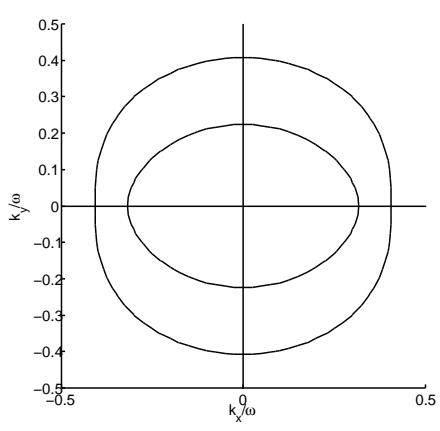

(f) MC.

Figure 2: Slowness diagrams.

the computational domain and therefore is not perfectly matched. In numerical experiments we have also observed that long-time solutions of the M-PML model may exhibit growth as the solutions reach quiescent state.

\subsection{Our perfectly matched layer}

In this section, we will derive the PML equations using the complex coordinate stretching technique, see $[6,17]$. The idea is to introduce new coordinates defined by special complex metrics. The basic properties of a PML can be found in [6]. The PML can be viewed as the complex change of variables in the Fourier transformed wave equation. We begin by 
taking Fourier transform in time,

$$
\begin{aligned}
& \mathbf{u}(x, y, t)=\int_{-\infty}^{\infty} \hat{\mathbf{u}}(x, y, \omega) e^{i \omega t} d \omega, \\
& -\omega^{2} \hat{\mathbf{u}}=A_{1} \hat{\mathbf{u}}_{x x}+A_{2} \hat{\mathbf{u}}_{y y}+A_{3} \hat{\mathbf{u}}_{x y} .
\end{aligned}
$$

To include PML in both $x$-direction and $y$-direction we follow the procedure: still in the frequency domain we introduce the complex metrics,

$$
s_{1}=\alpha_{1}(x)\left(1+\frac{\sigma_{1}(x)}{\eta+i \omega}\right), \quad s_{2}=\alpha_{2}(y)\left(1+\frac{\sigma_{2}(y)}{\eta+i \omega}\right)
$$

yielding

$$
-\omega^{2} \hat{\mathbf{u}}=A_{1} \frac{1}{s_{1}}\left(\frac{1}{s_{1}} \hat{\mathbf{u}}_{x}\right)_{x}+A_{2} \frac{1}{s_{2}}\left(\frac{1}{s_{2}} \hat{\mathbf{u}}_{y}\right)_{y}+A_{3} \frac{1}{s_{1}} \frac{1}{s_{2}} \hat{\mathbf{u}}_{x y} .
$$

Here, $\eta \geq 0$ is the complex frequency shift, $\alpha_{1}, \alpha_{2}>0$, are grid compression (or stretching) funtions and $\sigma_{1}, \sigma_{2}>0$ are the damping functions. The functions $\alpha_{1}, \alpha_{2}, \sigma_{1}, \sigma_{2}$ are also required to be smooth.

By choosing the auxiliary variables, and inverting the Fourier transform we have the full PML formulation

$$
\begin{aligned}
\mathbf{u}_{t t} & =\frac{1}{\alpha_{1}} A_{1}\left(\frac{1}{\alpha_{1}} \mathbf{u}_{x}\right)_{x}+\frac{1}{\alpha_{2}} A_{2}\left(\frac{1}{\alpha_{2}} \mathbf{u}_{y}\right)_{y}+\frac{1}{\alpha_{1} \alpha_{2}} A_{3} \mathbf{u}_{x y} \\
& -\frac{1}{\alpha_{1}} A_{1}\left(\frac{\sigma_{1}}{\alpha_{2}} \mathbf{v}-\frac{\sigma_{2}}{\alpha_{1}} \mathbf{w}\right)_{x}+\frac{1}{\alpha_{2}} A_{2}\left(\frac{\sigma_{1}}{\alpha_{2}} \mathbf{q}-\frac{\sigma_{2}}{\alpha_{1}} \mathbf{p}\right)_{y}-\left(\sigma_{1}+\sigma_{2}\right) \mathbf{u}_{t} \\
& +\left(\sigma_{1}+\sigma_{2}\right) \eta(\mathbf{u}-\mathbf{r})-\sigma_{1} \sigma_{2}((\mathbf{u}-\mathbf{r})-(\mathbf{r}-\mathbf{z})), \\
\mathbf{v}_{t} & =\frac{\alpha_{2}}{\alpha_{1}} u_{x}+\frac{\alpha_{2}}{\alpha_{1}} \sigma_{2} \mathbf{w}-\left(\sigma_{1}+\eta\right) \mathbf{v}, \\
\mathbf{w}_{t} & =\mathbf{u}_{x}-\eta \mathbf{w}, \\
\mathbf{p}_{t} & =\frac{\alpha_{1}}{\alpha_{2}} \mathbf{u}_{y}+\frac{\alpha_{1}}{\alpha_{2}} \sigma_{1} \mathbf{q}-\left(\sigma_{2}+\eta\right) \mathbf{p}, \\
\mathbf{q}_{t} & =\mathbf{u}_{y}-\eta \mathbf{q}, \\
\mathbf{r}_{t} & =\eta(\mathbf{u}-\mathbf{r}), \\
\mathbf{z}_{t} & =\eta(\mathbf{r}-\mathbf{z}) .
\end{aligned}
$$

In order to investigate some of the mathematical properties of the PML model (2.12) we will consider the vertical layer (parallel to the $y$-axis). We set $\sigma_{2}=0, \alpha_{2}=1 \mathrm{in}(2.12)$ and 
we have the reduced system

$$
\begin{aligned}
\mathbf{u}_{t t} & =\frac{1}{\alpha_{1}} A_{1}\left(\frac{1}{\alpha_{1}} \mathbf{u}_{x}\right)_{x}+A_{2} \mathbf{u}_{y y}+\frac{1}{\alpha_{1}} A_{3} \mathbf{u}_{x y} \\
& -\frac{1}{\alpha_{1}} A_{1}\left(\sigma_{1} \mathbf{v}\right)_{x}+A_{2}\left(\sigma_{1} \mathbf{w}\right)_{y}-\sigma_{1} \mathbf{u}_{t}+\eta \sigma_{1}(\mathbf{u}-\mathbf{r}), \\
\mathbf{v}_{t} & =\frac{1}{\alpha_{1}} \mathbf{u}_{x}-\left(\sigma_{1}+\eta\right) \mathbf{v}, \\
\mathbf{w}_{t} & =\mathbf{u}_{y}-\eta \mathbf{w}, \\
\mathbf{r}_{t} & =\eta(\mathbf{u}-\mathbf{r}) .
\end{aligned}
$$

By construction the model (2.13) is perfectly matched if $\sigma_{1}=0$ and $\alpha_{1}=1$ at the interface between the physical domain and the layer.

\subsection{Hyperbolicity and Well-posedness}

For convenience we shall re-write (2.13) as a first order system in time and space. Without loss of generality we consider $\eta=0, \alpha_{1}=1$, introduce the auxiliary variables,

$$
\begin{aligned}
& U_{1}=\mathbf{u}, \quad U_{2}=\mathbf{v}, \quad U_{3}=\mathbf{w}, \\
& U_{4}=\int_{0}^{t}\left(\mathbf{u}_{y}+\sigma_{1} \mathbf{w}\right) d \tau,
\end{aligned}
$$

and we have

$$
U_{t}=B_{x} U_{x}+B_{y} U_{y}+\sigma_{1} C U
$$

Here

$B_{x}=\left(\begin{array}{cccc}\mathbf{0} & A_{1} & A_{3} & \mathbf{0} \\ \mathbf{I} & \mathbf{0} & \mathbf{0} & \mathbf{0} \\ \mathbf{0} & \mathbf{0} & \mathbf{0} & \mathbf{0} \\ \mathbf{0} & \mathbf{0} & \mathbf{0} & \mathbf{0}\end{array}\right), B_{y}=\left(\begin{array}{cccc}\mathbf{0} & \mathbf{0} & \mathbf{0} & A_{2} \\ \mathbf{0} & \mathbf{0} & \mathbf{0} & \mathbf{0} \\ \mathbf{I} & \mathbf{0} & \mathbf{0} & \mathbf{0} \\ \mathbf{I} & \mathbf{0} & \mathbf{0} & \mathbf{0}\end{array}\right), C=\left(\begin{array}{cccc}-\mathbf{I} & \mathbf{0} & \mathbf{0} & \mathbf{0} \\ \mathbf{0} & \mathbf{- I} & \mathbf{0} & \mathbf{0} \\ \mathbf{0} & \mathbf{0} & \mathbf{0} & \mathbf{0} \\ \mathbf{0} & \mathbf{0} & \mathbf{I} & \mathbf{0}\end{array}\right)$,

where 0 is a $2 \times 2$ null matrix and $I$ is a $2 \times 2$ identity matrix.

It is easy to show that $\forall S=\left(S_{x}, S_{y}\right) \in \mathbb{R}^{2}$ normalized to satisfy (2.8), the matrix $\hat{B}=$ $S_{x} B_{x}+S_{y} B_{y}$ has real eigenvalues and a complete system of eigen-vectors. It follows that the PML model (2.13) is strongly hyperbolic, thus strongly well-posed, see [12]. However, because of the lower order term $\sigma_{1} C U$ the system (2.13) may have solutions that grow in time. It is important to note that strong hyperbolicity guarantees the well-posedness of partial differential equations under all lower order perturbations. The first order splitfield or modal PML formulations for the elastic wave equation are only weakly hyperbolic and lack this important property $[4,5]$. Including $\eta \neq 0$, will correspond to adding more zeros to the columns and rows of the matrices $B_{x}, B_{y}$ and the corresponding principal part is strongly hyperbolic. 


\section{Stability Analysis}

In this section we will explore the stability properties of the proposed layers. The perfectly matched layer is indeed a variable coefficient problem, but the mathematical tools readily available only allows for the analysis of the corresponding constant coefficient problem. Here we use standard perturbation techniques similar to the methods used in $[4,5,16]$, to analyze the corresponding constant coefficient Cauchy problem.

\subsection{Stability of the Standard PML}

To begin with, consider the model (2.13), we will show that at constant coefficients our layer suffers from the so called geometric (high frequency) instability. By introducing the modal ansatz $\mathbf{W}=\mathbf{W}_{0} e^{s t-i k_{x} x-i k_{y} y}$, where $\left(k_{x}, k_{y}\right)$ is the wave vector, and $\mathbf{W}=[\mathbf{u}, \mathbf{v}, \mathbf{w}, \mathbf{r}]^{T}$, we get the characteristic polynomial

$$
F\left(s, k_{x} / \alpha_{1}, k_{y}, \sigma_{1}, \eta\right) \equiv(s+\eta)^{2} F_{0}\left(\left(s+\sigma_{1}+\eta\right) s,(s+\eta) k_{x} / \alpha_{1},\left(s+\sigma_{1}+\eta\right) k_{y}\right)=0 .
$$

$F_{0}$ is defined in (2.6). The stability of the PML model (2.13) is characterized by the roots $s$ of the polynomial (3.1). In order to determine whether (2.13) is stable or unstable we only need to know the sign of the real part of $s, \Re s$ as we introduce damping.

First, we will characterize the roots of the undamped system. By setting $\sigma_{1}=0$ in (3.1) we have

$$
F\left(s, k_{x} / \alpha_{1}, k_{y}, 0, \eta\right)=(s+\eta)^{6} F_{0}\left(s, k_{x} / \alpha_{1}, k_{y}\right) .
$$

Clearly (3.2) has 10 roots, 4 of which are the purely imaginary $\Re s=0$ roots of (2.6) and correspond to the physical (quasi-P and quasi-S) modes. The remaining 6 roots are real $\Im s=0$ and strictly negative correspond to the non-physical modes,

$$
s_{m}=\{-\eta\}, \quad m=5,6, \ldots, 10 .
$$

We observe that the system (2.13) with $\left(\sigma_{1}=0\right)$ zero damping has no zero root for all $k_{x}, k_{y} \neq 0$. Since the roots depend continuously on the coefficients, it is apparent that at intermediate frequencies and with small enough damping the roots $s$ of the non-physical modes will remain in the left half of the complex plane. Thus we have

Lemma 1. At intermediate frequencies, if $\eta>0$ and $\alpha_{1}>0$, the non-physical modes $s$ are stable for all sufficiently small damping $\sigma_{1} \geq 0$.

At high frequencies a more refined analysis is needed.

Lemma 2. For any $\alpha_{1}>0$ and sufficiently small damping $\sigma_{1} \geq 0$ the parameter $\eta>0$ will stabilize the non-physical modes at high frequencies. 
Proof. We introduce the normalized variables,

$$
\lambda=\frac{s}{|k|}, \quad k_{1}=\frac{k_{x}}{|k|}, \quad k_{2}=\frac{k_{y}}{|k|}, \quad \epsilon=\frac{\sigma_{1}}{|k|}, \quad \gamma=\frac{\eta}{|k|}, \quad|k|=\sqrt{k_{x}^{2}+k_{y}^{2}},
$$

in (3.1) and we have

$$
(\lambda+\gamma)^{2} F_{0}\left((\lambda+\epsilon+\gamma) \lambda,(\lambda+\gamma) k_{1} / \alpha_{1},(\lambda+\epsilon+\gamma) k_{2}\right)=0
$$

We know that the non-physical modes are continuous functions of $\epsilon$ and therefore can be expanded by a Puiseux series, see [5],

$$
\lambda\left(k_{1} / \alpha_{1}, k_{2}, \epsilon\right)=-\gamma+Y^{\gamma}\left(k_{1} / \alpha_{1}, k_{2}\right) \epsilon^{r}+o\left(\epsilon^{r}\right), \quad r \in \mathbb{Q}^{+} .
$$

If $r \geq 1$, then we know that the perturbed root $\lambda$ will have a negative real part for sufficiently high frequencies and $\frac{\epsilon}{\gamma}<<1$, hence the non-physical modes are stable. Let us assume that there exists a solution such that $0<r<1$ and $Y^{\gamma} \neq 0$. By inserting the expression (3.6) in (3.5) we have

$$
\begin{gathered}
\left(\mathrm{Y}^{\gamma}\left(k_{1} / \alpha_{1}, k_{2}\right) \epsilon^{r}\right)^{6} F_{0}\left(-\gamma+O\left(\epsilon^{r}\right), k_{1} / \alpha_{1}+o(1), k_{2}+o(1)\right)=0, \\
\left.\Longrightarrow\left(\mathrm{Y}^{\gamma}\left(k_{1} / \alpha_{1}, k_{2}\right) \epsilon^{r}\right)^{6} F_{0}\left(-\gamma, k_{1} / \alpha_{1}, k_{2}\right)+o(1)\right)=0, \\
\Longrightarrow\left(\mathrm{Y}^{\gamma}\left(k_{1} / \alpha_{1}, k_{2}\right) \epsilon^{r}\right)^{6} F_{0}\left(-\gamma, k_{1} / \alpha_{1}, k_{2}\right)=0 .
\end{gathered}
$$

Since $F_{0}\left(-\gamma, k_{1} / \alpha_{1}, k_{2}\right) \neq 0$ for all $\gamma \in \mathbb{R}^{+}$, it follows that $Y^{\gamma}=0$, which is a contradiction. We must have $r \geq 1$ and the lemma is shown.

We note that the proof of Lemma 2 is analogous to the proofs of Lemma 7 in [5] and Theorem 1 in [4].

We see that if $\eta \neq 0$ the instability in the standard PML at constant coefficients can not come from the non-physical modes. In order to understand the stability of the physical modes we perform the high frequency stability analysis due to [4]. The physical modes are simple (distinct), therefore can be expanded in the powers of $\epsilon$,

$$
\lambda=\lambda_{0}+\epsilon \lambda_{\epsilon}+O\left(\epsilon^{2}\right) .
$$

Here $\lambda_{0}$ is purely imaginary. At sufficiently high frequencies, $\gamma, \epsilon<<1$, we can ignore higher order terms in $\gamma, \epsilon$, and the sign of the real part of $\lambda_{\epsilon}$ determines the stability of (2.13).

Consider

$$
F_{0}\left(\lambda,\left(1+\frac{\epsilon}{\lambda+\gamma}\right)^{-1} k_{1}, k_{2}\right)=0,
$$

and expand in the powers of $\epsilon, \gamma$ and we have

$$
F_{0}\left(\lambda_{0}, k_{1}, k_{2}\right)+\epsilon \lambda_{\epsilon} \frac{\partial F_{0}}{\partial \lambda}-\epsilon \frac{k_{1}}{\lambda_{0}} \frac{\partial F_{0}}{\partial k_{1}}+\gamma \frac{k_{1}}{\left|\lambda_{0}\right|^{2}} \frac{\partial F_{0}}{\partial k_{1}}+O\left(\epsilon^{2}+\gamma^{2}\right)=0
$$


By ignoring higher order terms and knowing that $\lambda_{0}, \partial F_{0} / \partial \lambda$ are purely imaginary, we have

$$
\Re \lambda_{\epsilon}=-\frac{k_{1}}{\lambda_{0}}\left(-\frac{\partial F_{0}}{\partial \lambda}\right)^{-1} \frac{\partial F_{0}}{\partial k_{1}}
$$

We see that the physical modes will be unstable if

$$
\frac{k_{1}}{\lambda_{0}}\left(-\frac{\partial F_{0}}{\partial \lambda}\right)^{-1} \frac{\partial F_{0}}{\partial k_{1}}<0
$$

The relation (3.9) is the so called geometric (high frequency) stability condition, Definition 1 . We have proved the following

Lemma 3. Consider $\alpha_{1}=1$ and $\sigma_{1}>0$. If the geometric stability condition in the $x$-direction is violated there are unstable physical modes at sufficiently high frequencies.

By computing the corresponding spectrum we also observed that increasing the complex frequency shift $\eta$ for a fixed $\sigma_{1}$ can move most of the unstable physical modes into the stable complex plane. However if $\eta>>\sigma_{1}$ the whole spectrum move towards the imaginary axis, indicating weak damping.

\subsection{Grid compression and high-frequency analysis}

Here we investigate the effect of the grid compression parameter $\alpha_{1}$ on the physical modes. We set $\gamma=\tilde{k} \epsilon$, where $\tilde{k}>0$. We use Maple to compute

$$
\lambda_{\epsilon}=-\frac{\partial F_{0}}{\partial \epsilon} /\left.\frac{\partial F_{0}}{\partial \lambda}\right|_{\epsilon=0}
$$

by implicit differentiation of (3.5). The result is

$$
\lambda_{\epsilon}=-\frac{f_{1} \alpha_{1}^{4}+f_{2} \alpha_{1}^{2}+f_{3}}{g_{1} \alpha_{1}^{4}+g_{2} \alpha_{1}^{2}+g_{3}} .
$$

Where

$$
\begin{aligned}
& f_{1}=-(2+3 \tilde{k})(b+c) k_{2}^{2}\left|\lambda_{0}\right|^{2}+(2+3 \tilde{k})\left|\lambda_{0}\right|^{4}+(2+3 \tilde{k}) b c k_{2}^{4}, \\
& f_{2}=\left(a b+c^{2}-d^{2}\right)(1+3 \tilde{k}) k_{1}^{2} k_{2}^{2}-(1+3 \tilde{k})(a+c) k_{1}^{2}\left|\lambda_{0}\right|^{2}, \\
& f_{3}=3 a c \tilde{k} k_{1}^{4}
\end{aligned}
$$

and

$$
\begin{aligned}
& g_{1}=-4(b+c) k_{2}^{2}\left|\lambda_{0}\right|^{2}+5\left|\lambda_{0}\right|^{4}+3 b c k_{2}^{4}, \\
& g_{2}=3\left(a b+c^{2}-d^{2}\right) k_{1}^{2} k_{2}^{2}-4(a+c) k_{1}^{2}\left|\lambda_{0}\right|^{2}, \\
& g_{3}=3 a c k_{1}^{4} .
\end{aligned}
$$


Qualitatively, if we fix $k_{1}, k_{2}$ and let $\alpha_{1} \rightarrow 0$ we see that, $\lambda_{\epsilon} \rightarrow \frac{f_{3}}{g_{3}}=-\tilde{k}<0$.

More precisely, for any $k_{0}>0$, if we choose $\alpha_{0}=k_{0} /((2+3 \tilde{k})(1+\max (1,|a|,|b|,|c|,|d|)))$, and $\tilde{k}>0$, we have

$$
\lambda_{\epsilon} \leq-\frac{f_{3}}{2 g_{3}}=\frac{-\tilde{k}}{2}<0 .
$$

for all $\left|k_{0}\right| \leq\left|k_{1}\right| \leq 1$ and $0 \leq\left|k_{2}\right| \leq 1$. This proves

Lemma 4. Given $\eta, \sigma_{1}, k_{0}>0$ there exists $\alpha_{0}>0$ such that for all $0<\alpha_{1} \leq \alpha_{0}$ the physical modes with the normalized frequencies $\left(k_{1}, k_{2}\right)$ in the range $\left|k_{0}\right| \leq\left|k_{1}\right| \leq 1,0 \leq\left|k_{2}\right| \leq 1$, are stable at sufficiently high frequencies.

A direct consequence of Lemmas 2 and 4 is the corollary

Corollary 1. For sufficiently high frequencies $|k| \rightarrow \infty$, if $\alpha_{1}, \eta$ satisfy the conditions in Lemmas 2 and 4, then the constant coefficient PML model (2.13) is asymptotically stable for all relevant frequencies, $\left|k_{0}\right|<\left|k_{1}\right| \leq 1,0 \leq\left|k_{2}\right| \leq 1$.

In order to highlight the above result, we consider two materials, Apatite and Zinc crystal violating the geometric stability condition, see Figures 3(a) and 3(c). These figures are obtained by considering a discrete set of frequencies $\left(k_{x}, k_{y}\right)$. In a discrete setting we expect a vertical layer to be unstable at least if the modes violating the stability condition are well resolved.

As we introduce grid compression, $\alpha_{1}=1 / 7$ for Apatite, and $\alpha_{1}=1 / 8.9$ for Zinc crystal, the slowness diagrams change see Figures 3(b) and 3(d), if we use thesame set of frequencies. This shows that on a fixed grid, grid compression can be used to avoid resolving the unstable modes.

In the next subsection we demonstrate that unresolved modes are much more stable than resolved modes.

\subsection{Semi-discrete analysis}

In the discrete setting, a finite number of grid points are used and derivatives are also approximated (by finite differences). For a given discretization the instability or stability in the continuous model can be strengthened or weakened. Here we demonstrate for the chosen discretization, the grid compression parameter $\alpha_{1}$ can be chosen such that the discrete PML is stable.

Replacing derivatives with standard second order central differences in (2.13), we have the semi-discrete problem

$$
\mathbf{u}_{t t}+\sigma_{1} \mathbf{u}_{t}=L_{h}(\mathbf{u}, W), \quad W_{t}=J_{h}(\mathbf{u}, W) .
$$

Here $W=(\mathbf{v}, \mathbf{w}, \mathbf{r})^{T}$, and $L_{h}, J_{h}$ are spatial discrete operators. 
We introduce the variable $\phi=\mathbf{u}_{t}$, to obtain a first order system in time and take discrete Fourier transform in space. Temporal stability is determined by the eigenvalue problem

$$
s \mathbf{U}=\tilde{\mathbf{D}}_{h} \mathbf{U} .
$$

Where

$$
\tilde{\mathbf{D}}_{h}=\left(\begin{array}{ccccc}
\mathbf{0} & \mathbf{I} & \mathbf{0} & \mathbf{0} & \mathbf{0} \\
D_{h}+\eta \sigma_{1} \mathbf{I} & -\sigma_{1} \mathbf{I} & \frac{i \sigma_{1}}{\alpha_{1} h} A_{1} \sin \left(h k_{x}\right) & \frac{i \sigma_{1}}{h} A_{2} \sin \left(h k_{y}\right) & -\eta \sigma_{1} \mathbf{I} \\
-\frac{i}{\alpha_{1} h} \sin \left(h k_{x}\right) \mathbf{I} & \mathbf{0} & -\left(\eta+\sigma_{1}\right) \mathbf{I} & \mathbf{0} & \mathbf{0} \\
\frac{i}{h} \sin \left(h k_{y}\right) \mathbf{I} & \mathbf{0} & \mathbf{0} & -\eta \mathbf{I} & \mathbf{0} \\
\eta \mathbf{I} & \mathbf{0} & \mathbf{0} & \mathbf{0} & -\eta \mathbf{I}
\end{array}\right),
$$

and

$$
D_{h}=-\frac{4}{\left(\alpha_{1} h\right)^{2}} A_{1} \sin ^{2}\left(h k_{x} / 2\right)-\frac{4}{h^{2}} A_{2} \sin ^{2}\left(h k_{y} / 2\right)-\frac{1}{\alpha_{1} h^{2}} A_{3} \sin \left(h k_{x}\right) \sin \left(h k_{y}\right) .
$$

is the (discrete) Fourier transform of the discrete approximations of the right-handside of (2.3). To investigate the stability of a numerical approximation at a certain resolution we compute the eigenvalues of $\tilde{\mathbf{D}}_{h}$ for the same spatial frequencies as were used for the slowness diagrams, Figures 3(b) and 3(d). Computations of the eigenvalues of $\tilde{\mathbf{D}}_{h}$ indicate that the semi-discrete problem is stable when the discrete slowness diagram does not violate the geometric stability condition. In fact we have observed that for the semi-discrete problem to be stable, it suffices to use an $\alpha_{1}$ so that the unstable modes are poorly resolved. This shows that the semi-discrete PML (3.13) has more robust stability properties than the corresponding continuous PML (2.13). However, as we refine the mesh, unstable modes appear. These unstable modes can also be removed by reducing $\alpha_{1}$ further.We conclude that if the grid is chosen such that the unstable modes are not well resolved, the discrete PML will be stable. 


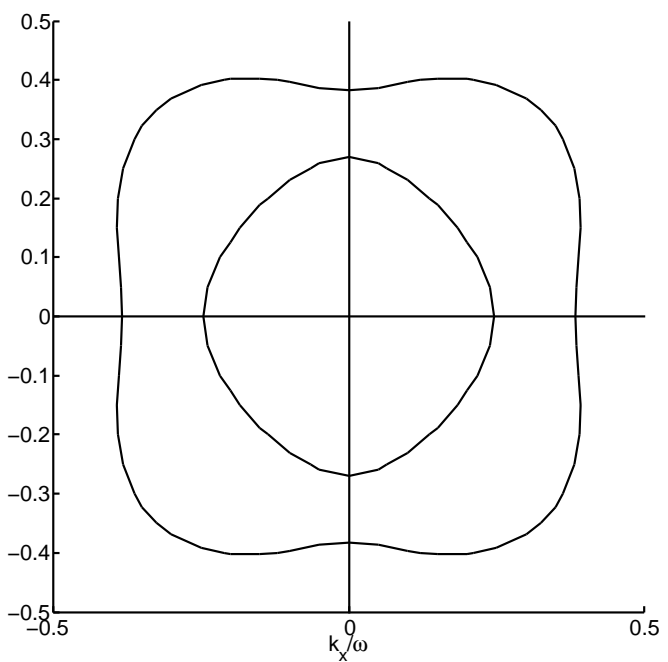

(a) Slowness diagram of Apatite

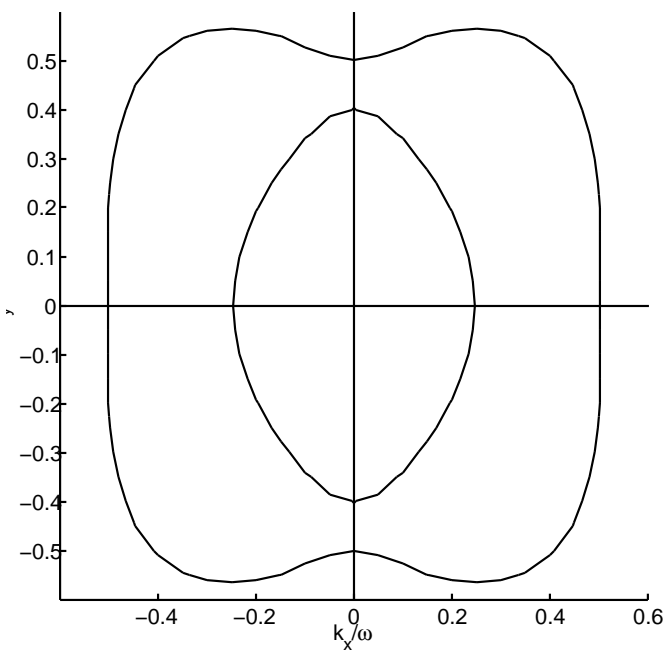

(c) Slowness diagram of Zinc crystal

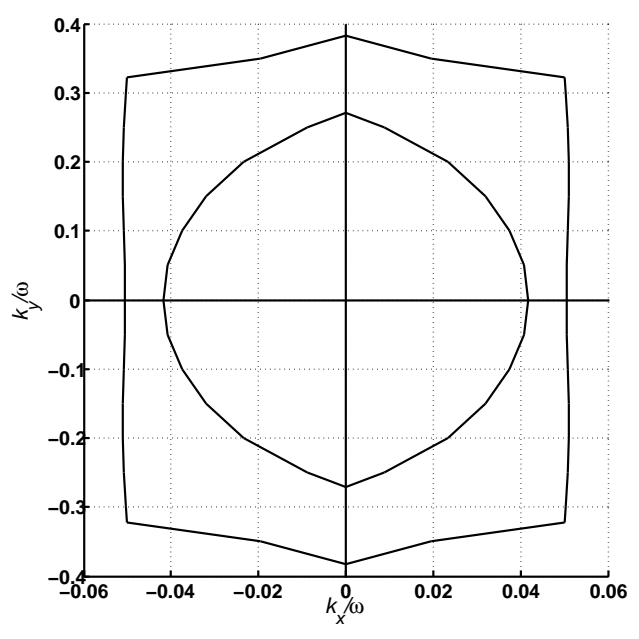

(b) Slowness diagram of Apatite, with $\alpha=1 / 7$

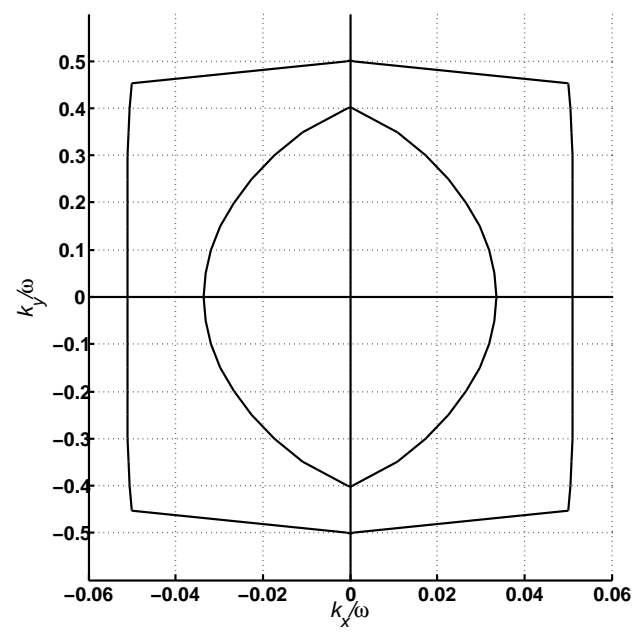

(d) Slowness diagram of Zinc crystal, with $\alpha=$ $1 / 8.9$

Figure 3: Slowness diagrams. 


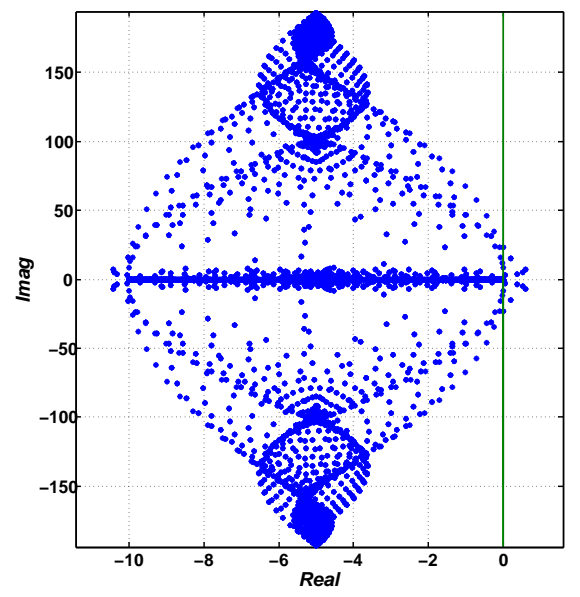

(a) MA2 (Apatite) $\eta=0, \alpha_{1}=1, \sigma_{1}=10$

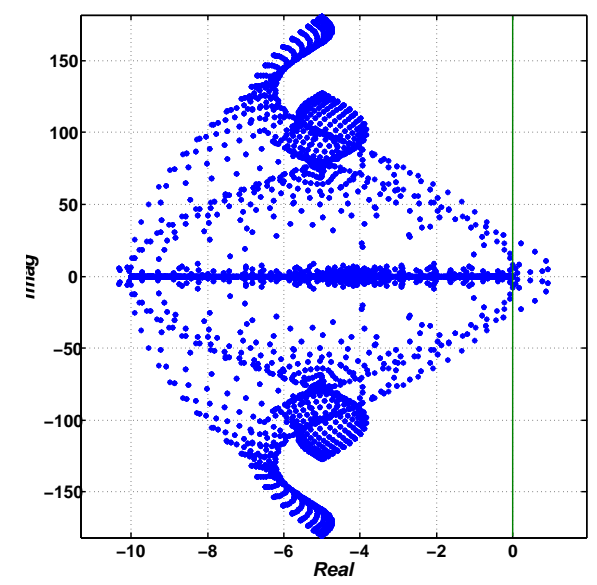

(c) MA4 (Zinc crystal) $\eta=0, \alpha_{1}=1, \sigma_{1}=10$. (d) MA4 (Zinc crystal) $\eta=5, \alpha_{1}=0.55, \sigma_{1}=$ 10 .

Figure 4: The discrete spectrum of the vertical layer (parallel to the $y$-axis) for Materials MA2 (Apatite) and MA4 (Zinc crystal)

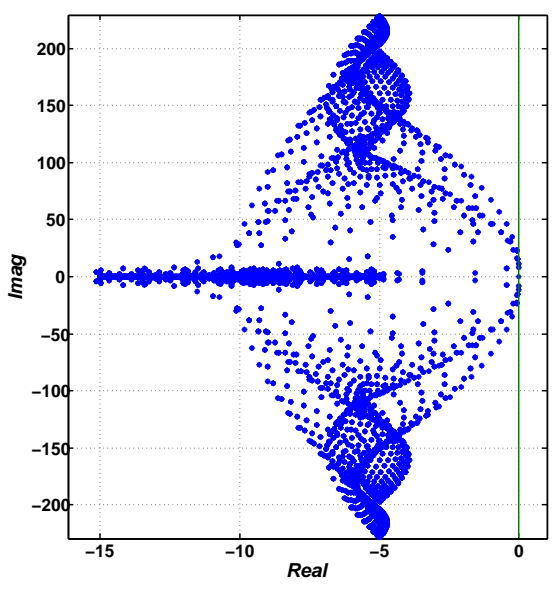

(b) MA2 (Apatite) $\eta=5, \alpha_{1}=0.8, \sigma_{1}=10$.

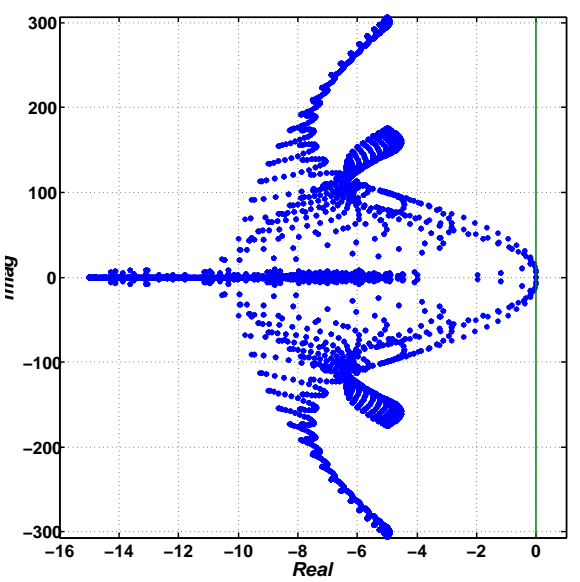




\subsection{Stability of the corner region}

If a Cartesian grid is surrounded by perfectly matched layers, corner regions are introduced where both damping coefficients $\sigma_{1}, \sigma_{2}>0$. In order to understand the behavior of the entire scheme, it is useful to investigate the stability of this corner region. In this section we show that the full PML formulation (2.12) is asymptotically stable. We know that in the continuous setting at sufficiently high frequencies the the complex frequency shift $\eta$ stabilizes the non-physical modes but does not guarantee the stability of the physical modes. We therefore consider the standard PML with $\eta=0, \alpha_{1}=\alpha_{2}=1$, (3.15) and investigate the stability of the physical modes in the corner region.

$$
\begin{aligned}
\mathbf{u}_{t t} & =A_{1} \mathbf{u}_{x x}+A_{2} \mathbf{u}_{y y}+A_{3} \mathbf{u}_{x y} \\
& -A_{1}\left(\sigma_{1} \mathbf{v}-\sigma_{2} \mathbf{w}\right)_{x}+A_{2}\left(\sigma_{1} \mathbf{q}-\sigma_{2} \mathbf{p}\right)_{y}-\left(\sigma_{1}+\sigma_{2}\right) \mathbf{u}_{t}-\sigma_{1} \sigma_{2} \mathbf{u}, \\
\mathbf{v}_{t} & =u_{x}+\sigma_{2} \mathbf{w}-\sigma_{1} \mathbf{v}, \\
\mathbf{w}_{t} & =\mathbf{u}_{x} \\
\mathbf{p}_{t} & =\mathbf{u}_{y}+\sigma_{1} \mathbf{q}-\sigma_{2} \mathbf{p}, \\
\mathbf{q}_{t} & =\mathbf{u}_{y} .
\end{aligned}
$$

We will prove the following Lemma

Lemma 5. Consider the constant coefficient PML (3.15) with $\sigma_{1}=\sigma_{2}=\sigma>0$, at sufficiently high frequencies $|k| \rightarrow \infty$, the physical modes are stable.

Proof. By assuming a modal solution $\mathbf{W}=\mathbf{W}_{0} e^{s t-i k_{x} x-i k_{y} y}$, and introducing the normalization (3.4) we have the eigenvalue problem,

$$
\lambda \mathbf{W}_{0}=D\left(k_{1}, k_{2}, \epsilon\right) \mathbf{W}_{0} .
$$

We know that at $\epsilon=0$, the physical modes are simple roots, we therefore consider the expansion (3.7) and study

$$
\lambda_{\epsilon}=\left.\frac{\partial \lambda}{\partial \epsilon}\right|_{\epsilon=0} .
$$

The characteristics polynomial corresponding to (3.16) is defined by

$$
F_{1}\left(\lambda, k_{1}, k_{2}, \epsilon\right)=\operatorname{det}\left(\mathbf{I} \lambda-D\left(k_{1}, k_{2}, \epsilon\right)\right) .
$$

With the symbolic mathematical software Maple we compute $\lambda_{\epsilon}$ by implicitly differentiation of $F_{1}\left(\lambda, k_{1}, k_{2}, \epsilon\right)$ evaluated at $\epsilon=0$,

$$
\lambda_{\epsilon}=-\frac{\partial F_{1}}{\partial \epsilon} /\left.\frac{\partial F_{1}}{\partial \lambda}\right|_{\epsilon=0}
$$

and we have

$$
\lambda_{\epsilon}\left(k_{1}, k_{2}\right)=\frac{f\left(k_{1}, k_{2}\right)}{g\left(k_{1}, k_{2}\right)}
$$


Where

$$
\begin{aligned}
f\left(k_{1}, k_{2}\right)= & -\left(4 \lambda^{4}+\left(3(a+c) k_{1}^{2}+(b+c) k_{2}^{2}\right) \lambda^{2}\right. \\
& +2\left(a c k_{1}^{4}+b c k_{2}^{4}+\left(a b+c^{2}-d^{2}\right) k_{1}^{2} k_{2}^{2}\right), \\
g\left(k_{1}, k_{2}\right)= & 6 \lambda^{4}+5\left((a+c) k_{1}^{2}+(b+c) k_{2}^{2}\right) \lambda^{2} \\
& +4\left(a c k_{1}^{4}+b c k_{2}^{4}+\left(a b+c^{2}-d^{2}\right) k_{1}^{2} k_{2}^{2}\right),
\end{aligned}
$$

At $\epsilon=0$, the physical modes satisfy the dispersion relation (2.6). Thus if we add

$$
-2\left(\lambda^{4}+\left((a+c) k_{1}^{2}+(b+c) k_{2}^{2}\right) \lambda^{2}+a c k_{1}^{4}+b c k_{2}^{4}+\left(a b+c^{2}-d^{2}\right) k_{1}^{2} k_{2}^{2}\right)=0,
$$

to $f\left(k_{1}, k_{2}\right)$, we have

$$
\lambda_{\epsilon}=-1<0
$$

The physical modes are stable for sufficiently small $\epsilon \geq 0$.

By the numerical computations of the eigenvalues of the symbol $D\left(k_{1}, k_{2}, \epsilon\right)$ we have found that at constant coefficients the PML (3.15) is also stable when $\sigma_{1} \neq \sigma_{2}$.

We expect all waves entering the corner region to decay exponentially in time and space. For a complete open domain problem, the corner regions enhance the stability of the entire scheme.

\section{Numerical Experiments}

In this section, we present some numerical experiments. The experiments aim at validating the theoretical results and further exploring the efficiency and stability properties of the proposed layers. Firstly, we shall briefly present the numerical method used. We will then discuss each problem set up, perform numerical experiments, present and discuss numerical results.

\subsection{Numerical method}

The numerical scheme is a node centered second order accurate (both in time and space) finite difference scheme. We will start with the spatial discretization and the time stepping scheme will follow. 


\subsubsection{Spatial discretization}

Let the grid in $(x, y)$ coordinates be defined by

$$
\begin{array}{ll}
x_{i}=i h_{x}, \quad i=0, \cdots, N_{x}-1, & h_{x}=1 /\left(N_{x}-1\right), \\
y_{j}=j h_{y}, \quad j=0, \cdots, N_{y}-1, & h_{y}=1 /\left(N_{y}-1\right) .
\end{array}
$$

We denote the grid function $\left[\mathbf{u}_{i, j}\right]$, the standard finite difference operators are denoted:

$$
\begin{aligned}
h_{x} D_{+}^{x} \mathbf{u}_{i, j} & =\mathbf{u}_{i, j+1}-\mathbf{u}_{i, j}, \quad h_{y} D_{+}^{y} \mathbf{u}_{i, j}=u_{i+1, j}-\mathbf{u}_{i, j}, \\
h_{x} D_{-}^{x} \mathbf{u}_{i, j} & =\mathbf{u}_{i, j}-\mathbf{u}_{i, j-1}, \quad h_{y} D_{-}^{y} \mathbf{u}_{i, j}=\mathbf{u}_{i, j}-\mathbf{u}_{i-1, j}, \\
2 D_{0}^{x} \mathbf{u}_{i, j} & =D_{+}^{x} \mathbf{u}_{i, j}+D_{-}^{x} \mathbf{u}_{i, j}, \quad 2 D_{0}^{y} \mathbf{u}_{i, j}=D_{+}^{y} \mathbf{u}_{i, j}+D_{-}^{y} \mathbf{u}_{i, j} .
\end{aligned}
$$

We also used averaging operators

$$
2 E_{1 / 2}^{x}\left(\sigma_{i, j}\right)=\sigma_{i, j+1}+\sigma_{i, j}, \quad 2 E_{1 / 2}^{y}\left(\sigma_{i, j}\right)=\sigma_{i+1, j}+\sigma_{i, j} .
$$

Replacing spatial derivatives with differences in (2.12), we have the semi-discrete problem

$$
\begin{aligned}
\mathbf{u}_{t t}+\left(\sigma_{1}+\sigma_{2}\right) \mathbf{u}_{t} & =L_{h}(\mathbf{u})+F_{h}\left(\mathbf{u}, \Theta, \sigma_{1}, \sigma_{2}, \eta\right), \\
\Theta_{t} & =J_{h}(\mathbf{u}, \Theta) .
\end{aligned}
$$

Where $\Theta=[\mathbf{v}, \mathbf{w}, \mathbf{p}, \mathbf{q}, \mathbf{r}, \mathbf{z}]^{T}, L_{h}, J_{h}$ are spatial discrete operators and the auxiliary function $F_{h}\left(\mathbf{u}, \Theta, \sigma_{1}, \sigma_{2}, \eta\right)$ ensures the perfect matching.

\subsubsection{Temporal Discretization}

We introduce the discrete time variable, $t_{n}=n d t, \quad n \in \mathbb{N}, d t$ is the time step and $\mathbf{u}\left(t_{n}\right) \approx \mathbf{u}^{n}$. The usual finite difference operators (with respect to time) are denoted

$$
d t^{2} D^{t t} \mathbf{u}^{n}=\mathbf{u}^{n+1}-2 \mathbf{u}^{n}+\mathbf{u}^{n-1}, \quad 2 d t D_{0}^{t} \mathbf{u}^{n}=\mathbf{u}^{n+1}-\mathbf{u}^{n-1}, \quad d t D_{+}^{t} \mathbf{u}^{n}=\mathbf{u}^{n+1}-\mathbf{u}^{n} .
$$

The time integration scheme uses, the leap-frog scheme for the physical variable and the Crank-Nicolson scheme for the auxiliary variables $(\Theta)$ and we obtain the full discrete PML problem

$$
\begin{aligned}
D^{t t} \mathbf{u}^{n}+\left(\sigma_{1}+\sigma_{2}\right) D_{0}^{t} \mathbf{u}^{n} & =L_{h}\left(\mathbf{u}^{n}\right)+F_{h}\left(\mathbf{u}^{n}, \Theta^{n}, \sigma_{1}, \sigma_{2}, \eta\right), \\
D_{+}^{t} \Theta^{n} & =\frac{1}{2} J_{h}\left(\mathbf{u}^{n+1}, \Theta^{n+1}\right)+\frac{1}{2} J_{h}\left(\mathbf{u}^{n}, \Theta^{n}\right) .
\end{aligned}
$$

If $\sigma_{1}=\sigma_{2}=0$, the discrete problem

$$
D^{t t} \mathbf{u}^{n}=L_{h}\left(\mathbf{u}^{n}\right),
$$

corresponding to the interior discretization of the elastic wave equation (2.3) can be shown to conserve discrete energy, [13]. 


\subsection{Stability of the PML}

Considering the strongly 'unstable' material MA1, we numerically study the stability of the second order PML model and the effect of the complex frequency shift $\eta$ for a monomial damping $\sigma_{1}(x)=(n+1) 5|x|^{n},(x, y) \in[-1,1]^{2}$ with the spatial step-size $h_{x}=$ $h_{y}=0.05$. We use the initial condition

$$
\begin{aligned}
& u_{1}=u_{2}=\exp \left(-\ln (2) \frac{x^{2}+y^{2}}{\delta}\right), \quad \delta=0.02, \\
& u_{1 t}=u_{2 t}=0 .
\end{aligned}
$$

for the displacement field and homogeneous initial data for the auxiliary variables. We set homogeneous Dirichlet boundary conditions in the $x$-direction and periodic boundary conditions in the $y$-direction.

As predicted by the analysis in the preceding section, at constant coefficients the PML solutions grow in time and growth depends on $\max \sigma_{1}$, see Figure $5(\mathrm{~d})$. The growth also remains for variable coefficients. With our choice of damping function the local values of $\sigma_{1}$ decreases with increasing degree of the monomial in a large part of the layer, and therefore it is not surprising that the growth rate decreases with increasing monomial degree, see Figures 6(b) and 5(b). However, with grid refinement this behaviour will probably change. In conclusion, for a given resolution, using higher order monomials seems advantagous as the damping power of the layer can be held constant by our choice of damping function while reducing the severity of the growth.

Theoretical analysis at constant coefficients predicts that the complex frequency shift stabilizes the non-physical modes. We see from Figure 6(b) and 5(b) that the introduction of the complex frequency shift reduces the growth rate signficantly. However, the absorption power of the PML for a fixed width layer is weakened.

From Figure 5(c), we see that growth rate increases with resolution, also the growth rate approaches a constant as we refine the mesh. The explanation is that if the mesh is such that the growing modes are not well resolved the discrete PML is stable. For material MA1 at constant coefficients, the observed growth is strong because this material MA1 severely violates the geometric stability condition, see Figure 2(a). We also note that if the violation of the geometric stability is mild (as in MA2, MA3, MA4), on a reasonably fine mesh the discrete PML will be stable, see Figure 6. 


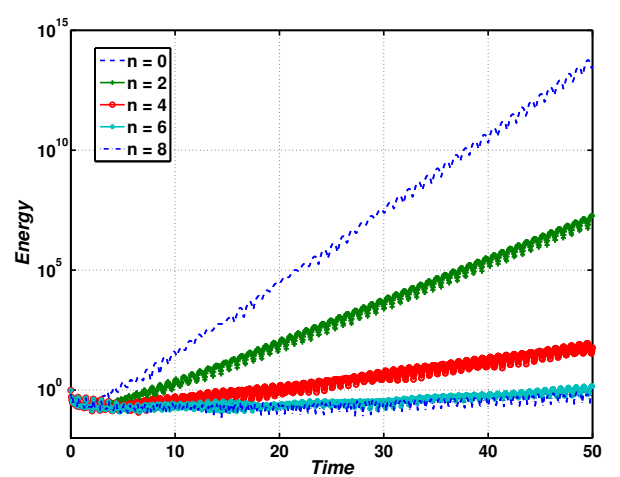

(a) Vertical layer $\eta=0$.

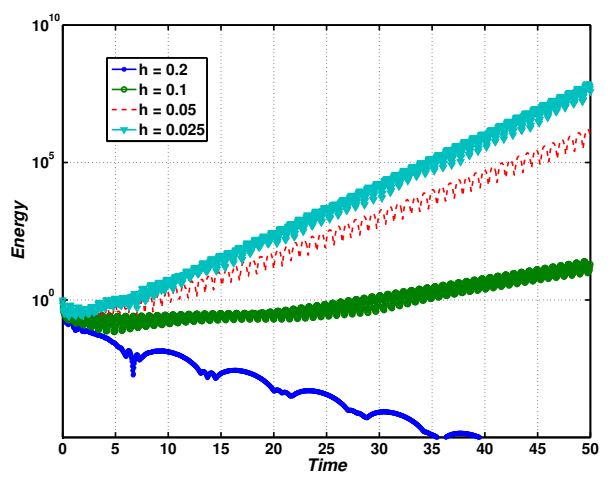

(c) Vertical layer $\eta=1, \sigma_{1}=5$.

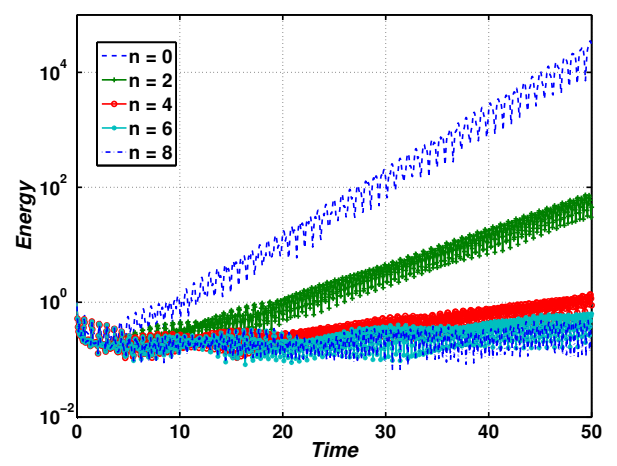

(b) Vertical layer $\eta=1+0.1 \sigma_{1}$.

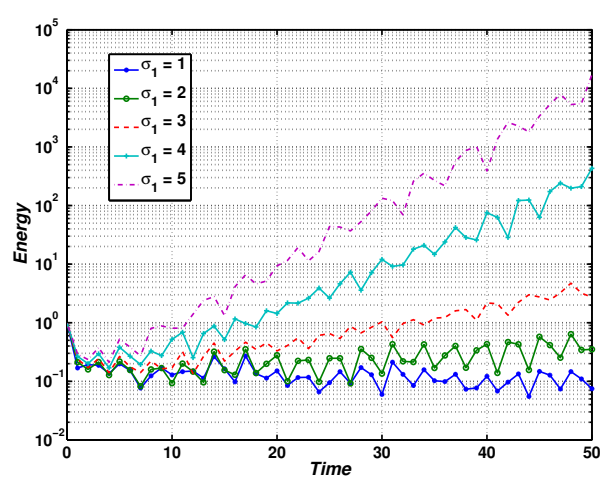

(d) Vertical layer $\eta=1+0.1 \sigma_{1}, \sigma_{1}=$ const.

Figure 5: The maximum elastic energy $\left(\left\|\sqrt{u_{1}^{2}+u_{2}^{2}}\right\|_{\infty}\right)$.

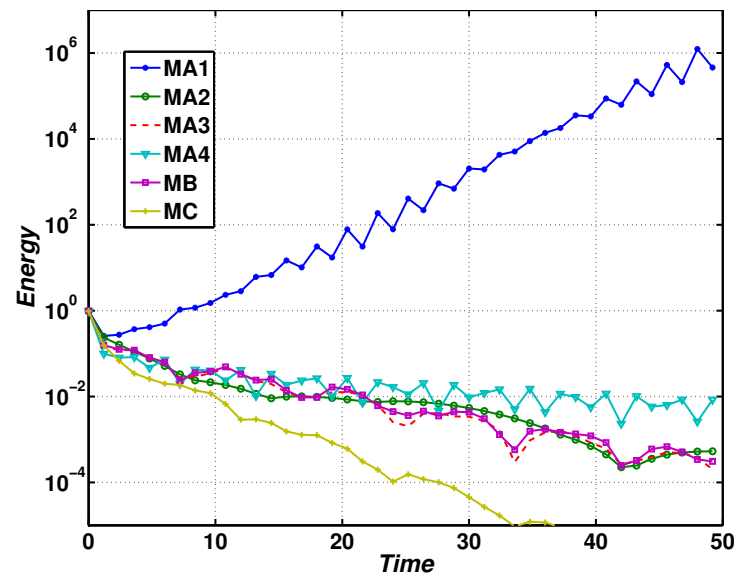

(a) Vertical layer $\eta=1, \sigma_{1}=5$

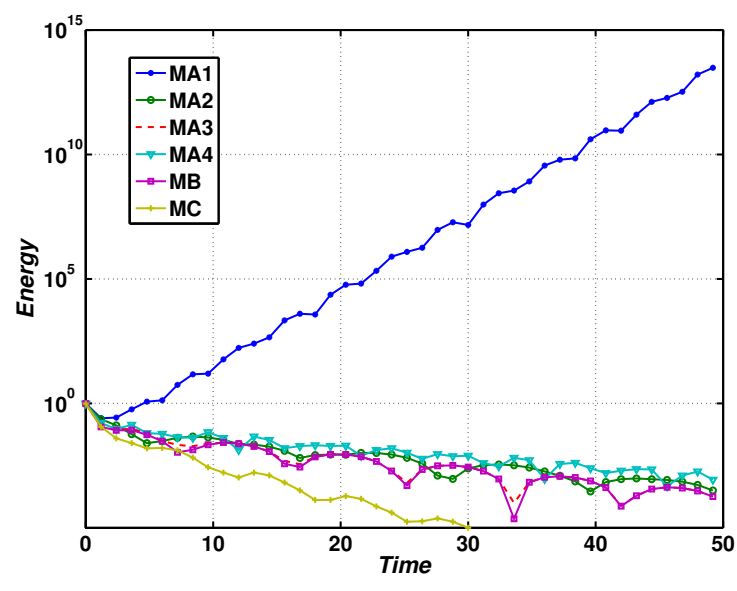

(b) Horizontal layer $\eta=1, \sigma_{1}=5$

Figure 6: The maximum elastic energy $\left(\left\|\sqrt{u_{1}^{2}+u_{2}^{2}}\right\|_{\infty}\right), h=0.05$. 


\subsection{Ducted problem}

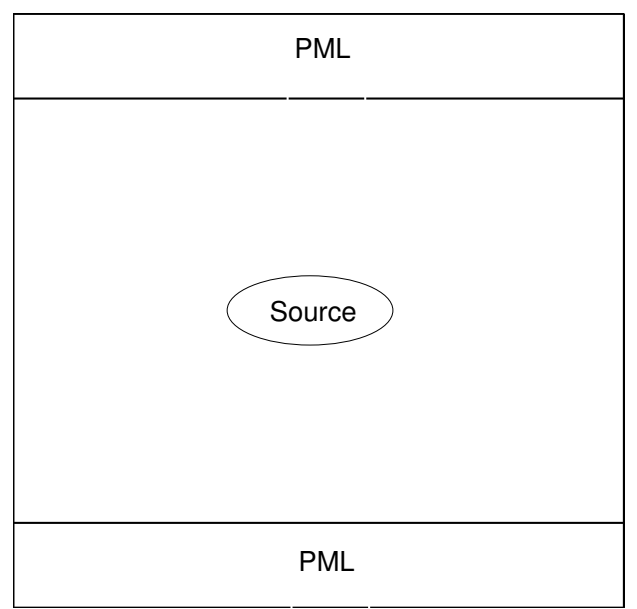

Figure 7: Computational domain

In this subsection we apply the PML to a ducted elastic wave problem. The physical domain is the infinite strip $-5 \leq x \leq 5,-\infty<y<\infty$. The problem is periodic in the $x$-direction but extends to the infinite space in the $y$-direction. However, in order to perform numerical experiments we truncate the domain in the $y$-direction such that the computational domain is a square $-5 \leq x \leq 5-5 \leq y \leq 5$. To simulate the infinite space, we add two additional layers, having $5 \leq|y| \leq 6$, which corresponds to $10 \%$ of the width of the computational domain as shown in Figure 7 , in which the PML equations are solved. For the displacement field we use the initial data

$$
\begin{aligned}
& u_{1}=u_{2}=\exp \left(-\ln (2) \frac{x^{2}+y^{2}}{\delta}\right), \quad \delta=0.2 \\
& u_{1 t}=u_{2 t}=0
\end{aligned}
$$

and homogeneous initial data for the auxiliary variables. In the $y$-direction we set homogeneous Dirichlet boundary conditions, and periodic boundary conditions in the $x$ direction, for all variables.

The damping profile is the monomial of the form

$$
\begin{aligned}
& \sigma_{2}(y)=\left\{\begin{array}{ccc}
0 & \text { if } & |y| \leq y_{0}, \\
d_{0}\left(\frac{|y|-y_{0}}{y_{1}-y_{0}}\right)^{n} & \text { if } & |y| \geq y_{0}
\end{array}\right. \\
& d_{0}>0, \quad n=4, \quad y_{0}=5, \quad y_{1}=6 .
\end{aligned}
$$


We choose $d_{0}=4.5(n+1) /\left(2\left(y_{1}-y_{0}\right)\right) \log (1 / \operatorname{Re} f)$ such that the reflections from the outer boundaries is of the magnitude Ref, see $[4,23]$. Here, 4.5 is the maximum wave speed for the elastic materials considered.

In order to highlight the effectiveness of the proposed layers we shall consider the difficult elastic material MA1 (and the strongly 'unstable' horizontal layer). From the slowness diagram Figure 2(a) we see that the material MA1 severely violates the geometric stability condition, and we expect the standard PML to be unstable.

We discretize the domain by introducing the uniform mesh-size $h_{x}=h_{y}=0.1$ such that the initial pulse is well resolved. We set $\operatorname{Re} f=10^{-3}$, introduced the complex frequency shift $\eta=1+0.1 \sigma_{2}$ and compute the solutions until $T=70.0$ The behavior of the PML is illustrated in Figure 8, showing how the initial pulse spreads, enters into the layer and it is being absorbed. After a long time $T=70$ the growing solutions corrupts the solution in the interior of the domain, see Figure 9(a) .

We coarsened the mesh $h_{x}=h_{y}=0.2$ and compute until $T=100$. There was no growing solutions, see Figure 9(b). From the numerical experiments performed the materials MA2, MA3, and MA4 where the violation of the geometric stability condition is mild, we have found that if the violations of the geometric stability condition are not severe, on a reasonably fine mesh the discrete PML is stable. We set the complex frequency shift $\eta=0$ the growing solutions reappear as shown in Figure 9(c), highlighting the stabilizing effect of the complex frequency shift. In order to further investigate the behavior of the layer we repeat the above experiment (with $\eta=0, h=0.1$ ) and zoom very close to the layer $((x, y) \in[-5,5] \times[5,6])$ as shown in Figures 10 and 11 . We carefully study the dynamics of the waves transmitted into the layer. We observe that the solutions in the layer are smooth and waves propagating normal to the boundary are perfectly absorbed. The remnant propagates tangentially unabsorbed in the layer. Because of the periodic boundary conditions in the tangential direction, with increasing time waves propagate back and forth in the layer and grow slowly, see Figures 10 and 11.

In order to make a comparison we apply the split-field PML $[4,23]$ to the first order (velocity-stress) formulation of the elastic wave equation. Considering the same set-up and resolution $h=0.1$, as above we discretized the equations using standard second order accurate centered finite difference approximations in space and standard second order Runge-Kutta method in time. We compute the solutions until a final time $T$. Since we are interested in the behavior of the layer we zoom very close to the layer $((x, y) \in[-5,5] \times$ $[5,6])$ as show in Figure 12. We see that as the slower $S$-wave penetrates the layer, at approximately $t=4.0$ the solution in the layer in this case grows destructively in time. However, as we coarsened the mesh the solutions remained unstable but with a slower growth rate. We believe the strong instability observed here and reported in $[4,5]$ is related to the high-frequency parasitic numerical modes that are present in the standard discretizations of the first order formulation. Analysis of the discrete behaviours of PML models is a topic of future work. 


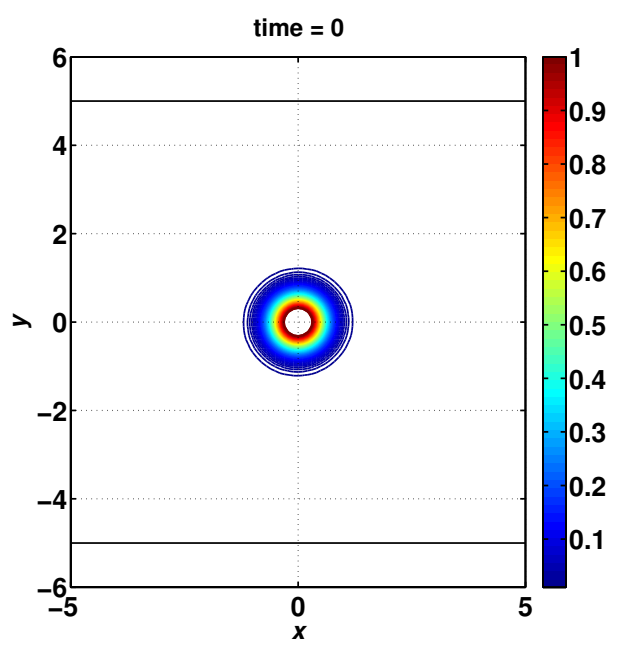

(a) $t=0.0$

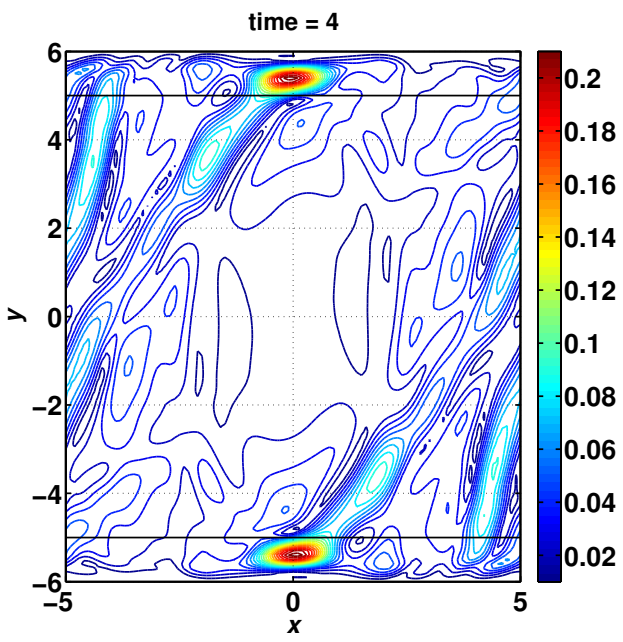

(c) $\mathrm{t}=4.0$

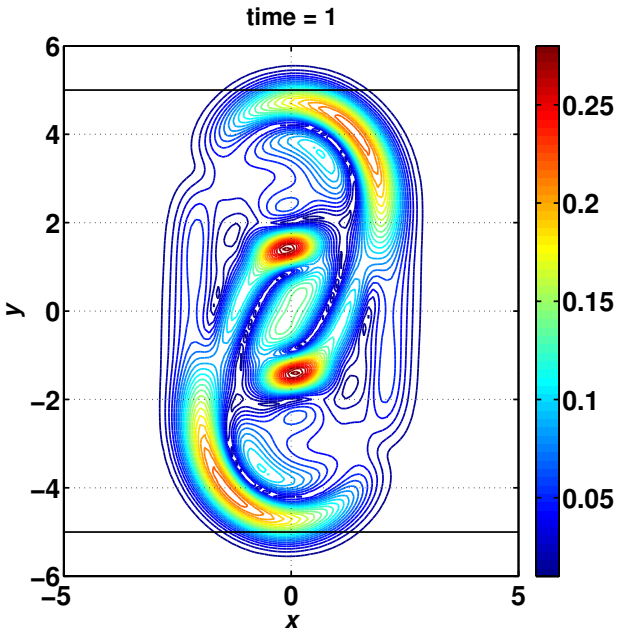

(b) $\mathrm{t}=1.0$

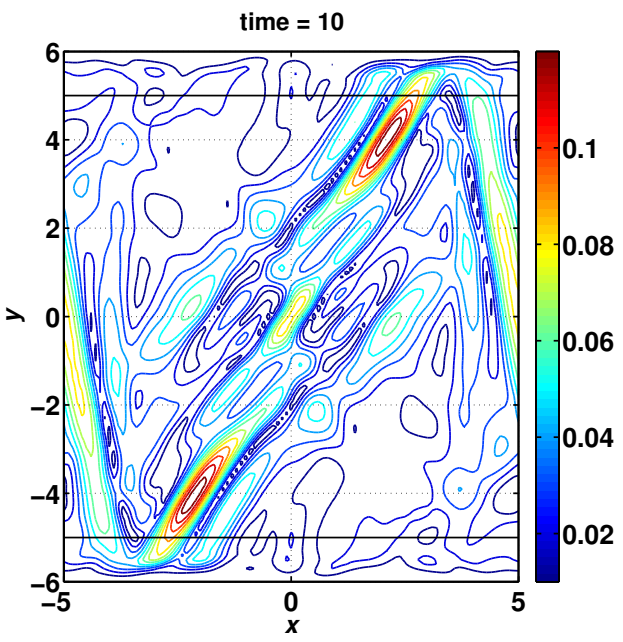

(d) $\mathrm{t}=10.0$

Figure 8: The dynamics of $\sqrt{u_{1}^{2}+u_{2}^{2}}$ for the new second order PML for the 'unstable' Material MA1.

\subsubsection{Grid compression}

From the analysis, we expect that for a given resolution, the introduction of a grid compression parameter will stabilize the physical modes. This idea was tested for material MA1 and the resolution $h_{x}=h_{y}=0.1$. For that case we observed growth, see from Figure 9(a). A grid compression $\alpha_{2}=1-0.5 \exp (-1 /(5(|y|-5))$ was introduced in the layer and it resulted to a stable solution, see Figure 9(d). However, as we refine the grid we expect the growth to reappear, the growth can also be removed by reducing the size of $\alpha_{2}$. The 


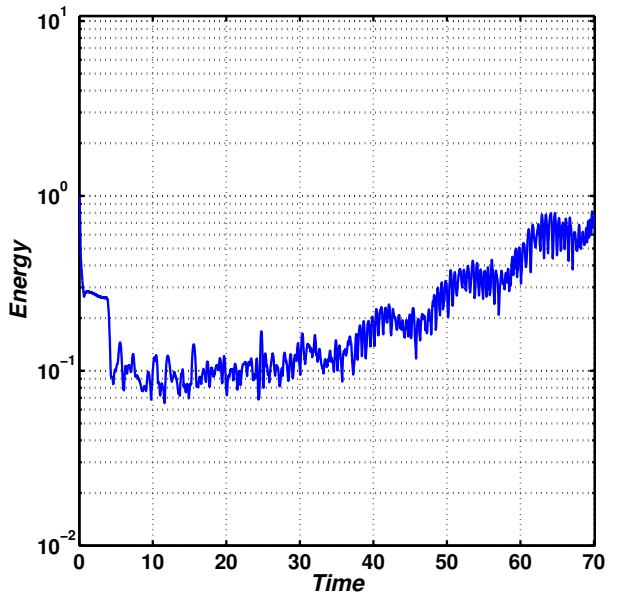

(a) $\eta=1+0.1 \sigma_{2}, h=0.1, \alpha_{2}=1$.

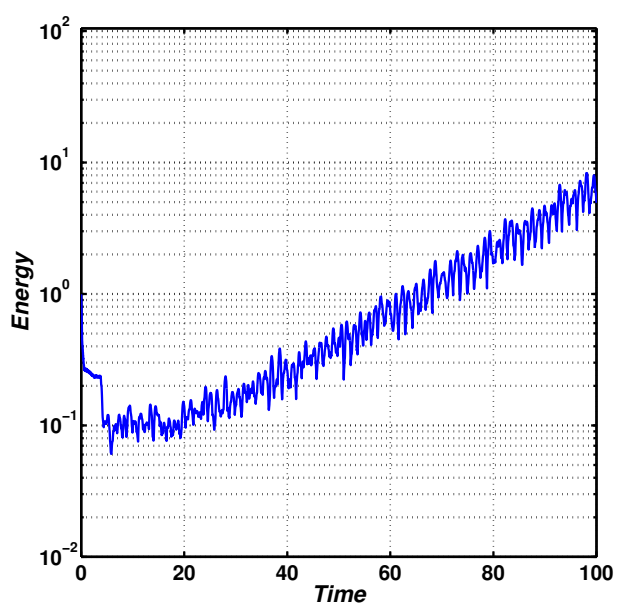

(c) $\eta=0.0, h=0.2, \alpha_{2}=1$.

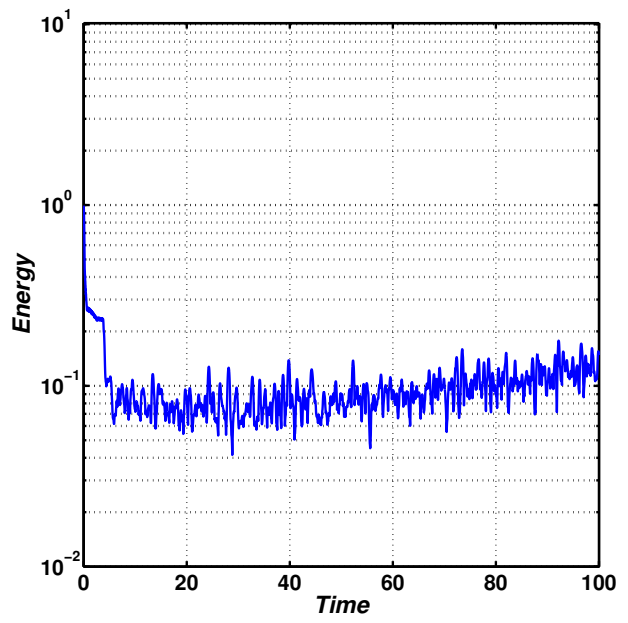

(b) $\eta=1+0.1 \sigma_{2}, h=0.2, \alpha_{2}=1$.

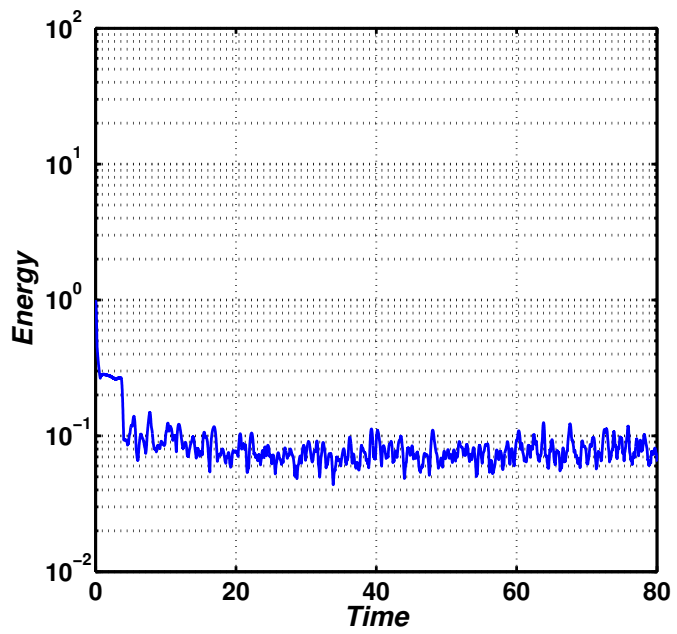

(d) $\eta=1+0.1 \sigma_{2}, \quad h=0.1, \quad \alpha_{2}=1-$ $0.5 \exp (-1 /(5(|y|-5))$

Figure 9: The maximum energy $\left\|\sqrt{u_{1}^{2}+u_{2}^{2}}\right\|_{\infty}$ in the interior of the domain.

drawback here is that the problem becomes stiff with decreasing $\alpha_{2}$ and will consequently restrict the time step.

However, it is important to mention that the materials MA2, MA3, and MA4 where the violation of the geometric stability condition is mild, no growth was observed at this resolution, and does not require any grid compression yet. We believe that this would probably change with finer resolutions. 


\subsection{Complete open-domain}

Here we consider a complete open domain problem. The computational domain consists of the rectangular two-dimensional domain $(x, y) \in[-10,10] \times[-10,10]$, surrounded by a PML of width 2 . The damping parameter $d_{0}=50$, and the complex frequency shift $\eta=1.0$. We force the first component of the displacement vector with

$$
\begin{aligned}
& F(x, y, t)=f(t) \frac{1}{\delta^{2}} e^{-7\left((x-8)^{2}+(y+8)^{2}\right) / \delta^{2}}, \\
& f(t)=\left(2 \pi^{2}(0.9 t-1)^{2}-1\right) e^{-\pi(0.9 t-1)^{2}}, \quad \delta=0.5 .
\end{aligned}
$$

An equidistant grid $h_{x}=h_{y}=0.125$ is used everywhere. We compute the solution until $T=50.0$. The behavior of the model is displayed in Figure 14 for material MA1 and in Figure 15 for material MA2, showing how the wave generated by the forcing $F(x, y, t)$ spreads, penetrates the PML and it is being absorbed. Figure 13, shows the time history of the maximum energy $\left\|\sqrt{u_{1}^{2}+u_{2}^{2}}\right\|_{\infty}$ in the interior of the computational domain, showing the stability of the layer and long time decay of the energy.

For material MA1, we observed a slowly growing solution in the layer $((x, y) \in[-12,12] \times$ $[10,12])$. But the growing solution enters the corner region $((x, y) \in[10,12] \times[10,12])$ and decays in time, (note the factor $10^{-3}$ in Figure 14). Furthermore, if we set the damping to a 6th degree monomial (for $h=0.125$ ) or coarsen the mesh $h=0.2$ (for the 4 th degree monomial), in these cases we did not observe any growth in the layers. For the material MA2 there was no growth observed with these resolutions, but with further refinement we may expect to see growth also for material MA2. 


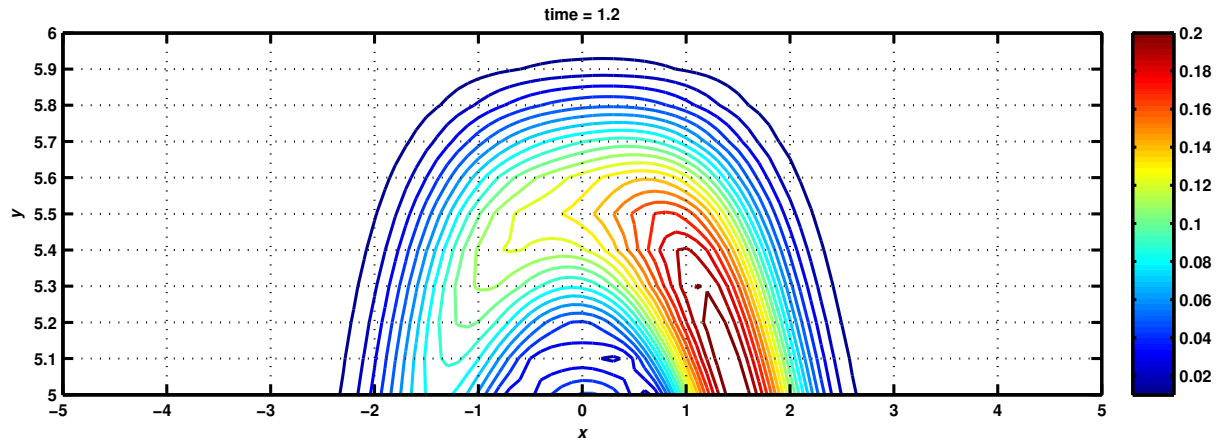

(a) $\mathrm{t}=1.2$

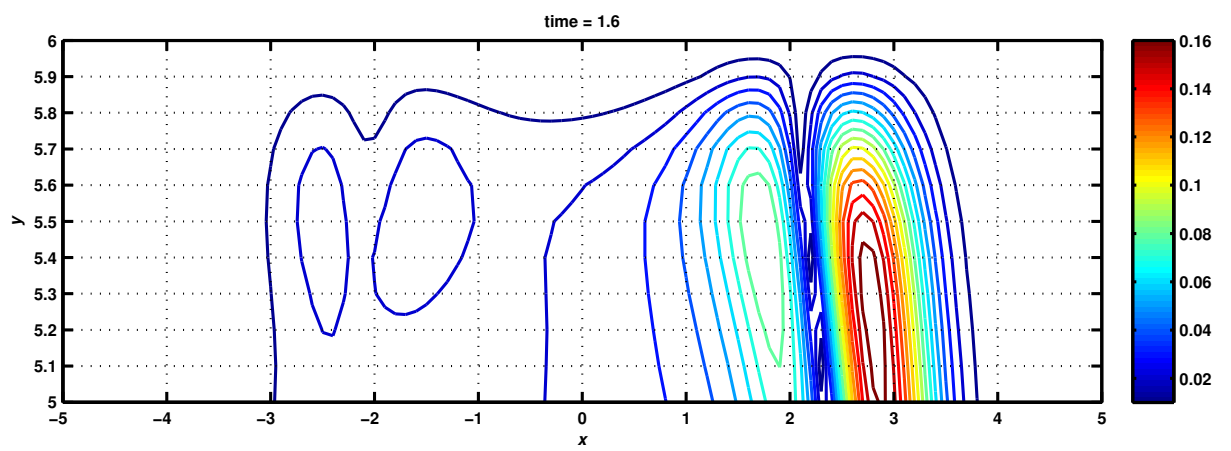

(b) $t=1.6$

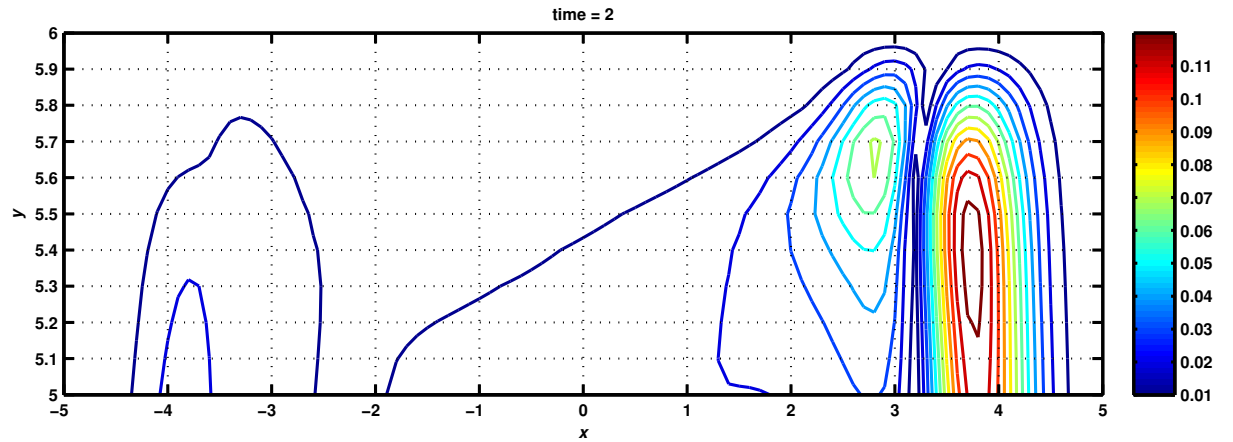

(c) $\mathrm{t}=2$

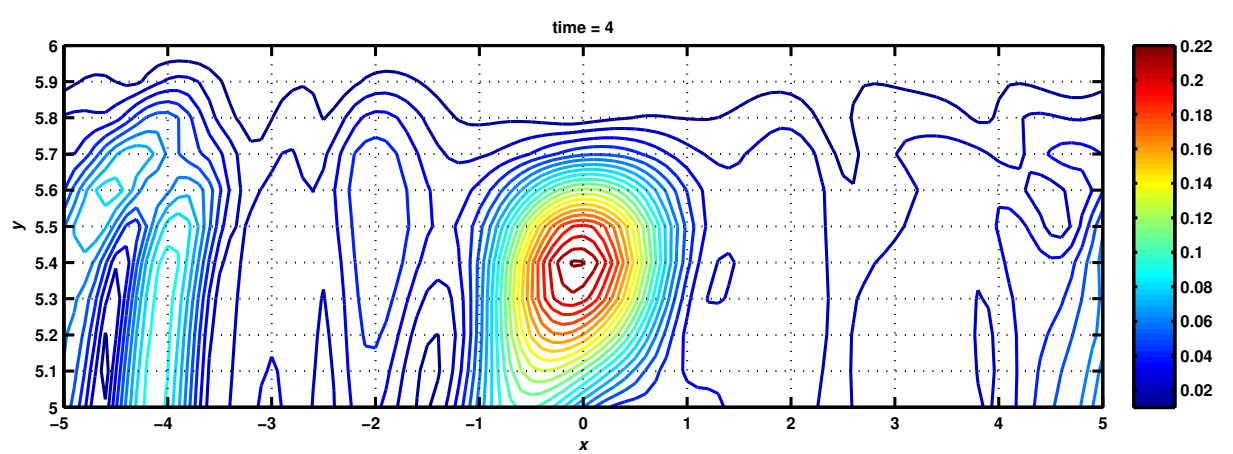

(d) $\mathrm{t}=4$

Figure 10: The dynamics of $\sqrt{u_{1}^{2}+u_{2}^{2}}$ inside the PML for the 'unstable' Material MA1. All figures are with contours between -1 and 1 at intervals of 0.01 , exempting the zero contour. 


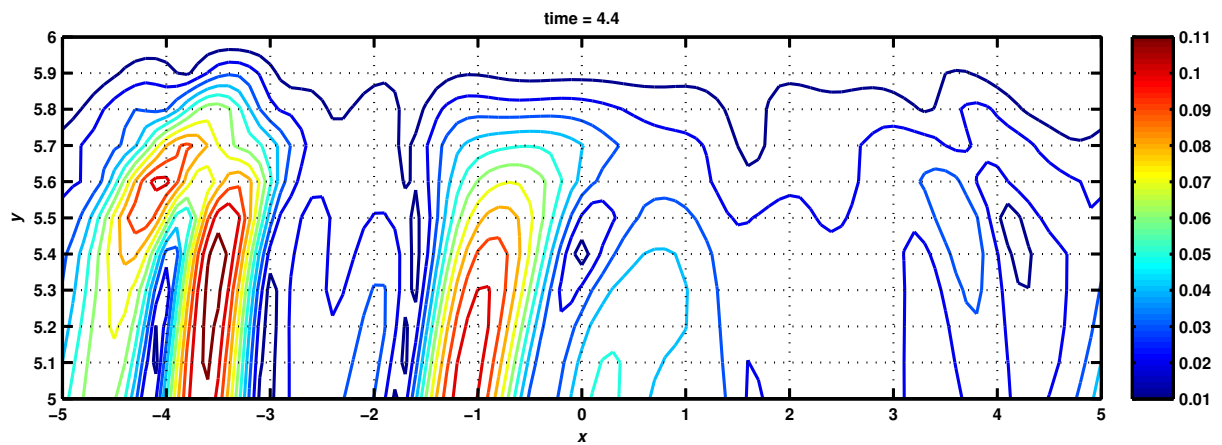

(a) $\mathrm{t}=4.4$

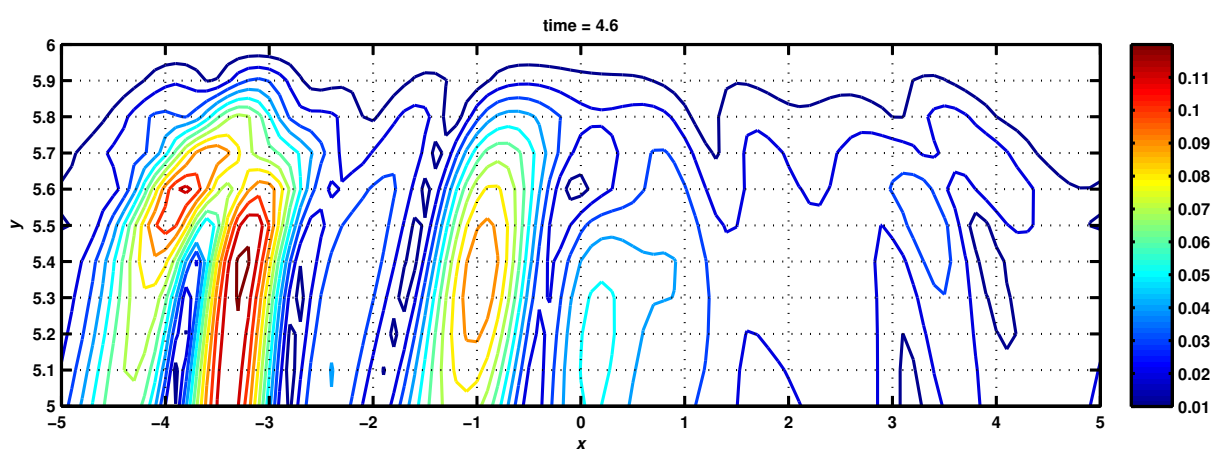

(b) $\mathrm{t}=4.6$

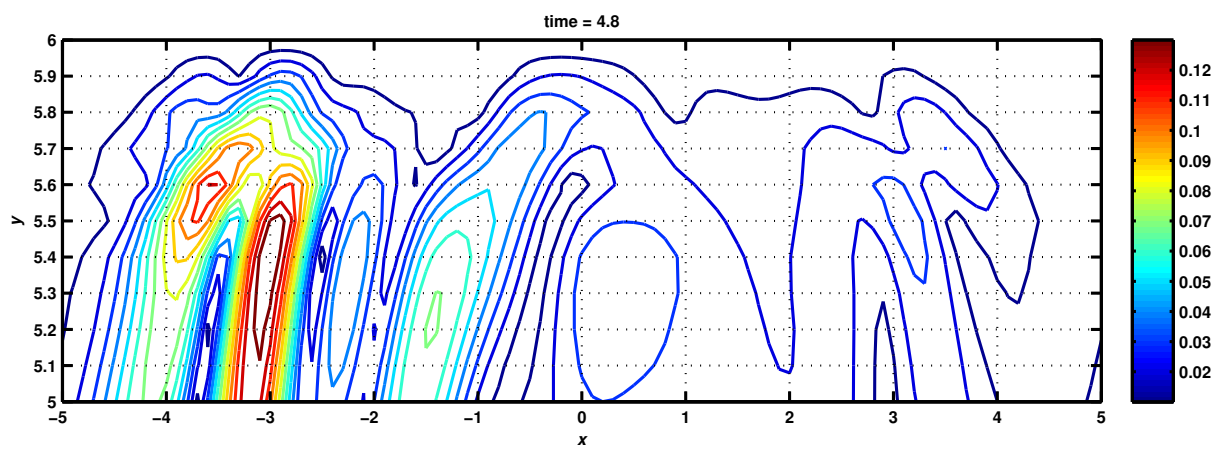

(c) $\mathrm{t}=4.8$

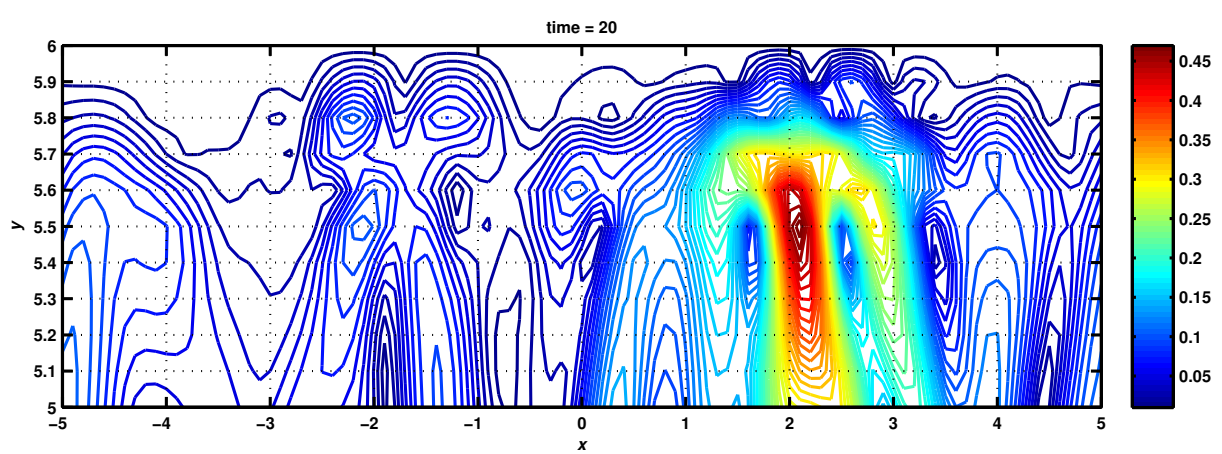

(d) $t=20$

Figure 11: The dynamics of $\sqrt{u_{1}^{2}+u_{2}^{2}}$ inside the PML for the 'unstable' Material MA1. All figures are with contours between -1 and 1 at intervals of 0.01 , exempting the zero contour. 


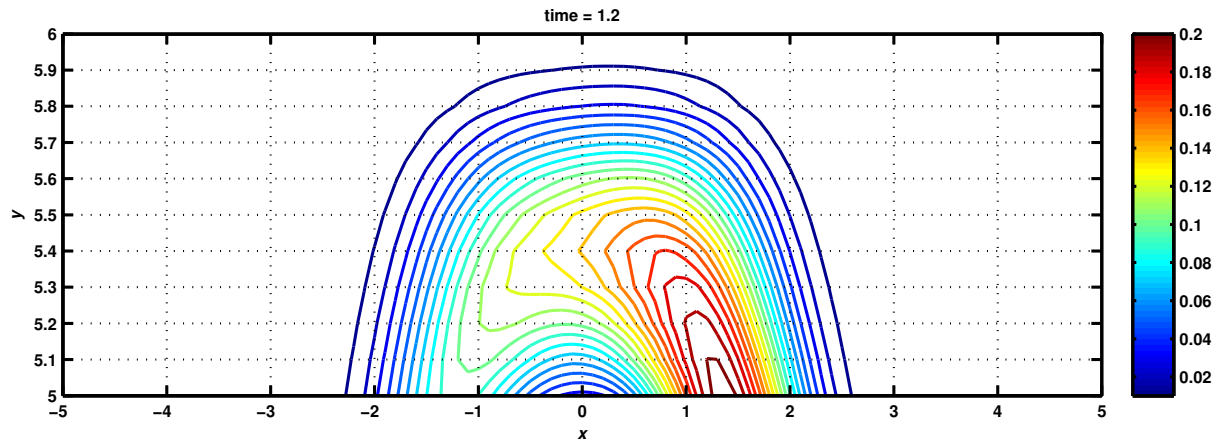

(a) $\mathrm{t}=1.2$

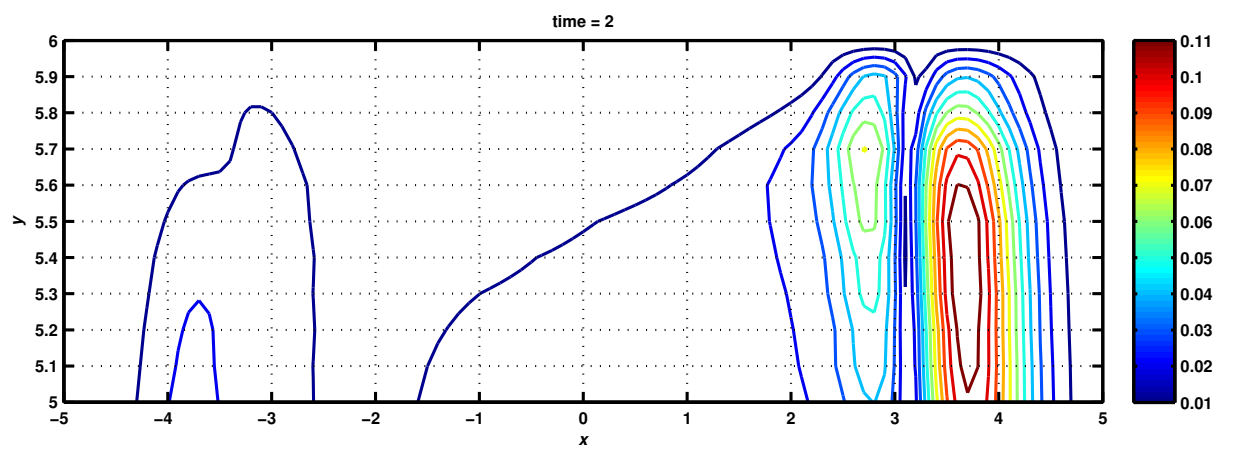

(b) $t=2$

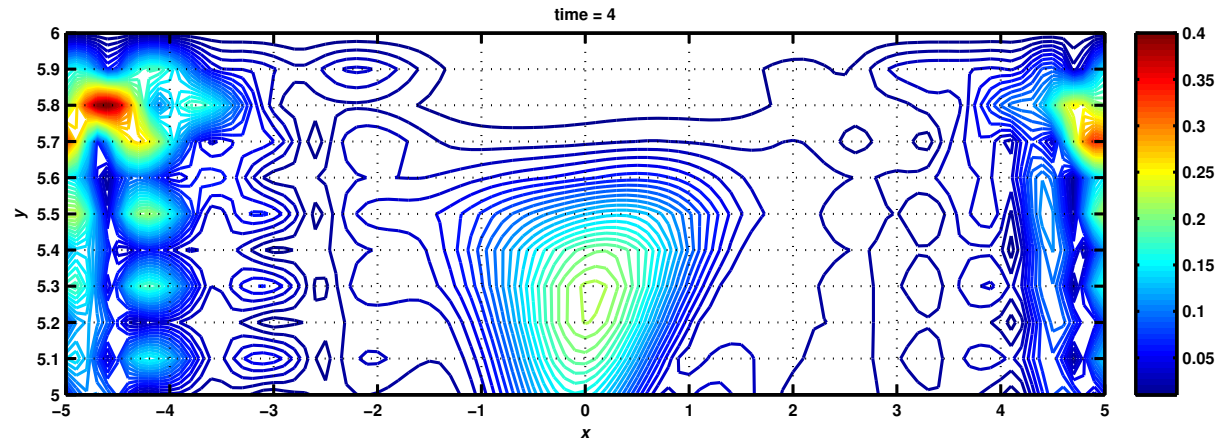

(c) $\mathrm{t}=4$

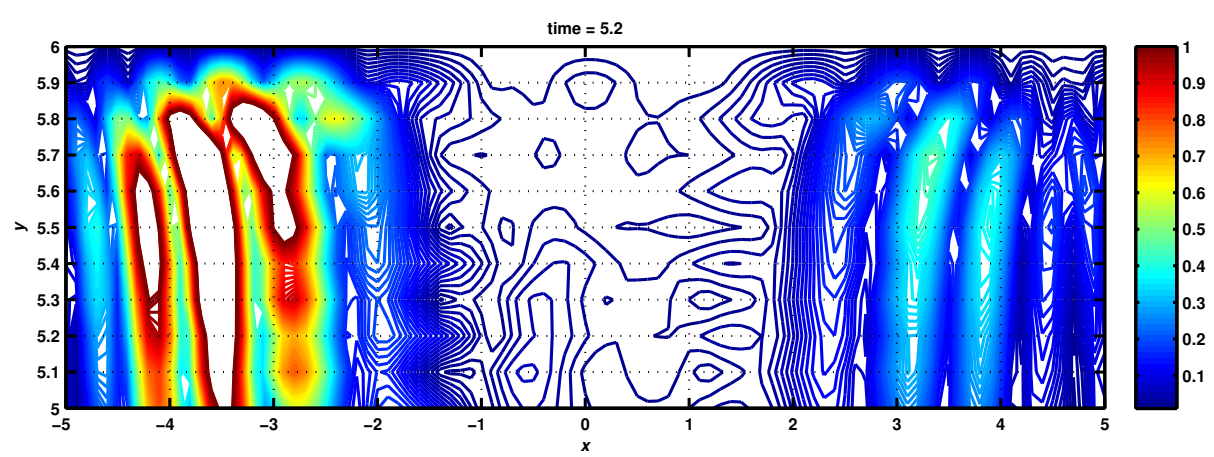

(d) $\mathrm{t}=5.2$

Figure 12: The dynamics of $\sqrt{u_{1}^{2}+u_{2}^{2}}$ inside the split-field PML for the 'unstable' Material MA1. All figures are with contours between -1 and 1 at intervals of 0.01 , exempting the zero contour. 


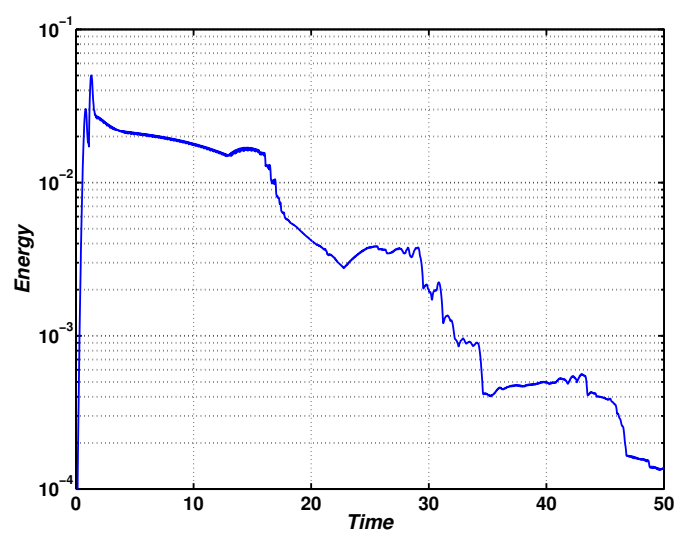

(a) MA1.

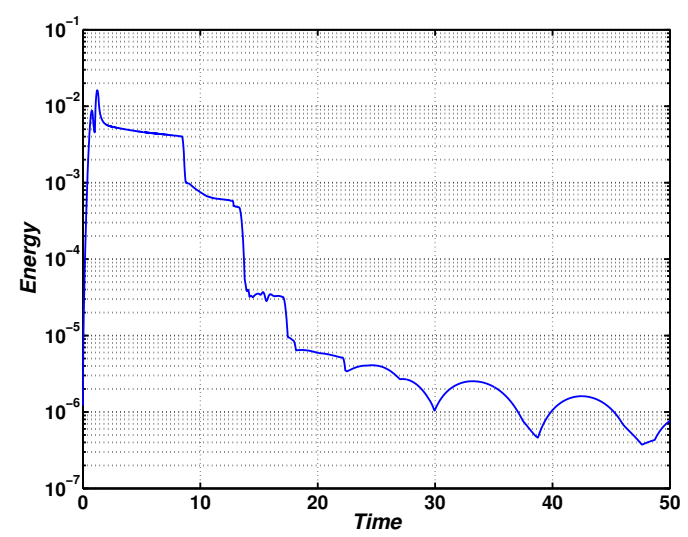

(b) MA2.

Figure 13: The maximum elastic energy $\left(\left\|\sqrt{u_{1}^{2}+u_{2}^{2}}\right\|_{\infty}\right)$ inside the computational domain, with $h=0.125, n=4$.

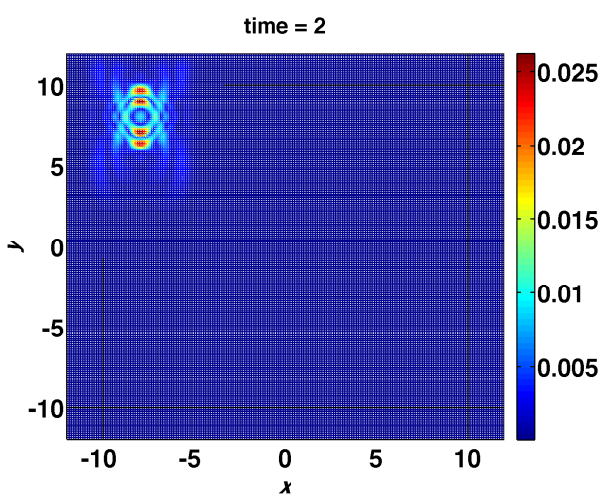

(a) $\mathrm{t}=2$.

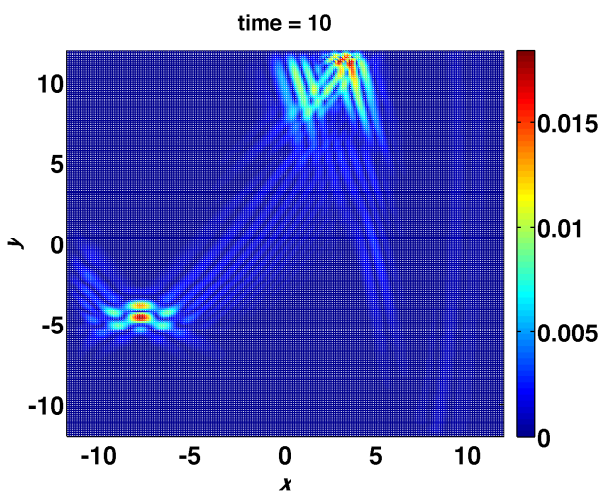

(c) $\mathrm{t}=10$.

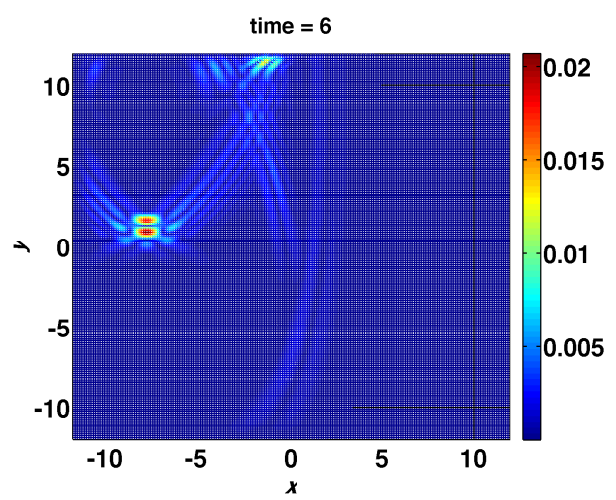

(b) $\mathrm{t}=6$.

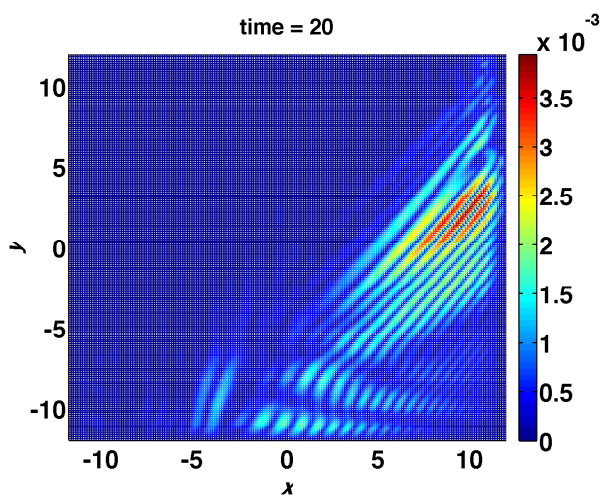

(d) $\mathrm{t}=20$.

Figure 14: The dynamics of $\sqrt{u_{1}^{2}+u_{2}^{2}}$ for MA1, with $h=0.125, n=4$. 


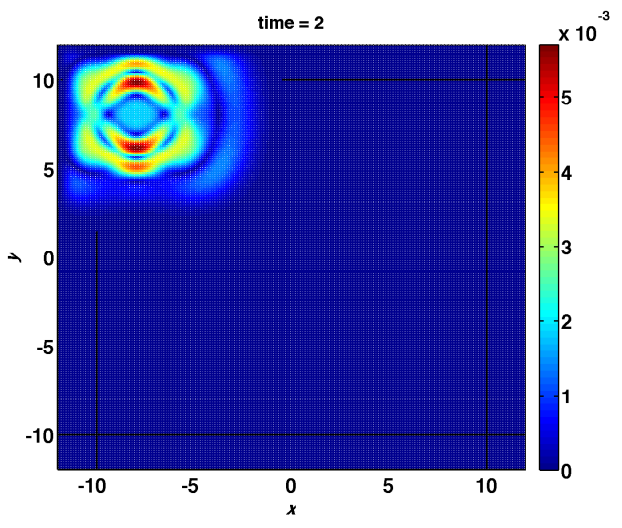

(a) $t=2$.

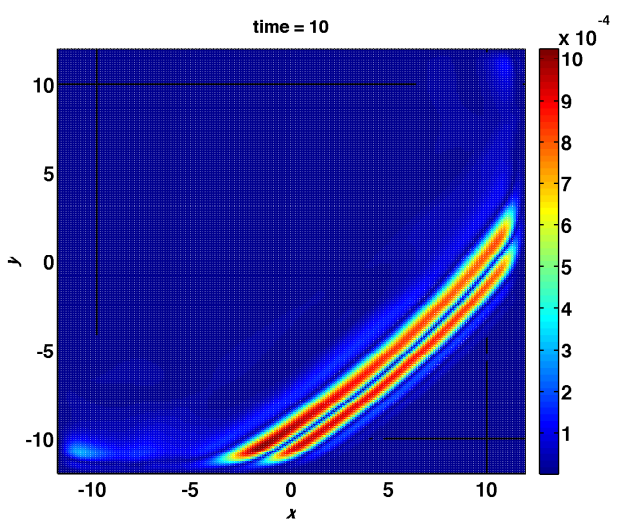

(c) $\mathrm{t}=10$.

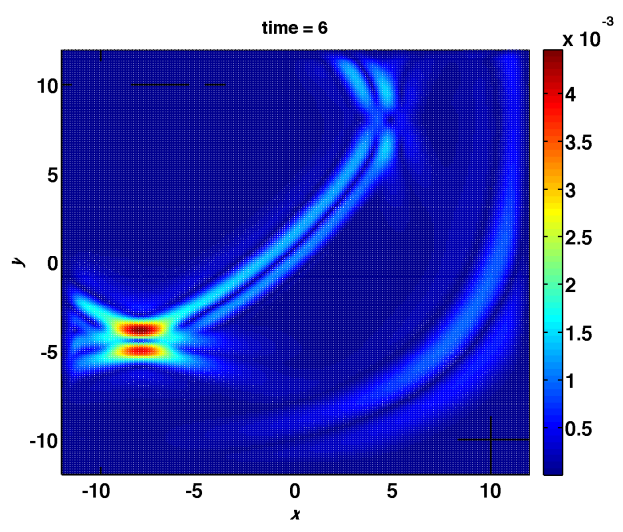

(b) $\mathrm{t}=6$.

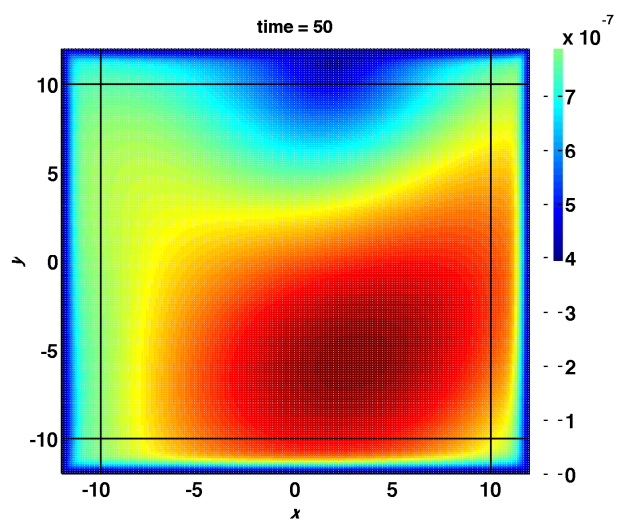

(d) $t=20$.

Figure 15: The dynamics of $\sqrt{u_{1}^{2}+u_{2}^{2}}$ for MA2, with $h=0.125, n=4$. 


\subsubsection{Convergence}

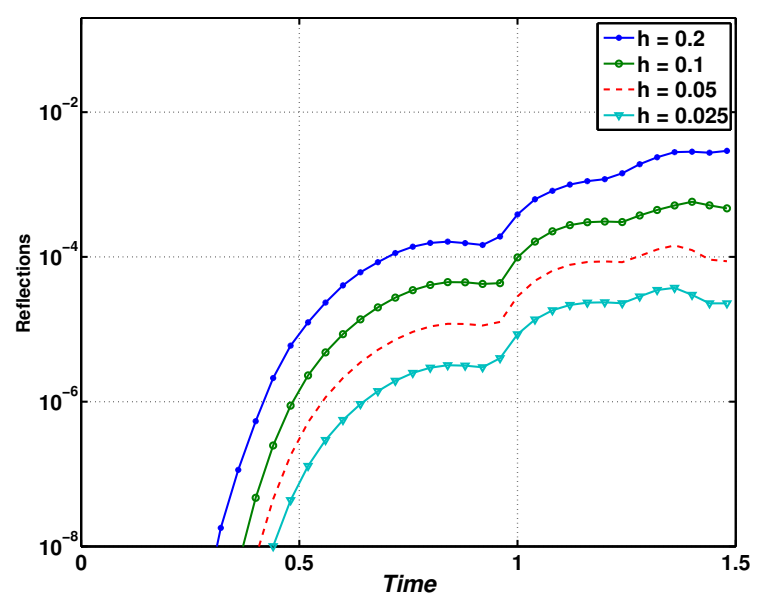

Figure 16: Numerical reflection

When a PML model is used in a numerical computation, errors can be divided into 3 different categories: the discretization error, numerical reflection, and the modeling error. The discretization error is the generic error that comes with any numerical method (by approximating derivatives by differences). numerical reflections, are the spurious discrete effects introduced by discretizing the PML and seen in the interior domain. The modeling error is introduced because the layer has a finite width. The discretization error and numerical reflection should vanish as the mesh-size approaches zero and the modeling error decreases as the magnitude of damping coefficient or the PML width increases.

Here we show that we can choose the PML parameters (for a fixed PML width) as a function of the grid-size such that the total PML error (modeling error + numerical reflection) converges at the same rate as the discretization error. We consider the elastic material MA1 and the domain $-d-2 \leq x \leq 2+d,-d-2 \leq y \leq 2+d$, where $d=2$ is the PML width. The initial data is same as (4.5) with $\delta=0.05$.

The damping profiles are monomials of degree 5 . We set $d_{0}=4.5(3) /(d) \log \left(1 /\left(C_{0} h^{2}\right)\right)$ so that numerical reflection from the interface will be small compared to to the modeling error. $C_{0}=0.01$ has been empirically determined. In this case the numerical scheme converges quadratically. Therefore the discretization error $\sim O\left(h^{2}\right)$, the modeling error $\sim$ $\exp \left(-\int \sigma d \xi\right)=O\left(h^{2}\right)$ and the numerical reflection $\sim O\left(d_{0} h^{2}\right)=O\left(h^{2}|\log (h)|\right)$. The total PML error is expected to approach zero at $\sim O\left(h^{2}|\log (h)|\right)$.

We compute the solution until $T=1.5$ so that the modeling error affects the solution in the interior. We a also compute a reference solution in a larger domain without the PML. By comparing the PML solution in the interior of the domain to the reference solution in the $\infty$-norm we obtain an accurate measure of the PML error. In Figure 16 we plot the time history of the PML error. The fast P-waves with velocity $C_{p}=4.5$ completely penetrate the layers at about $t=0.45$ and generates numerical reflection which level off 


\begin{tabular}{|l||l|l|}
\hline Mesh-size $(h)$ & Reflections & Rate \\
\hline \hline 0.2000 & $2.9000 \times 10^{-3}$ & - \\
0.1000 & $4.7060 \times 10^{-4}$ & 2.6326 \\
0.0500 & $8.7565 \times 10^{-5}$ & 2.4261 \\
0.0250 & $2.3856 \times 10^{-5}$ & 1.8760 \\
\hline
\end{tabular}

Table 2: Numerical reflections as a function of $h$

before $t=1$. At about $t \approx 1$ the slow S-waves with velocity $C_{s}=2.3$ penetrate the layers while the modeling error due to P-waves are simultaneously returning from the outer boundaries. At $t \geq 1$ the numerical reflection from the interface and modeling error from the outer boundaries superimpose, impact the solution in the interior but level off as time increases. However, from the choice of our damping coefficient we have almost quadratic convergence of the total PML error, see Figure 16 and Table 2.

Remark 1. We note that no growth was seen in the layer for these short-time computations. Since the continuous PML for material MA1 is unstable at sufficiently high frequencies, we expect late time growth in the layer for sufficiently small mesh-sizes. If the damping in the corner region is not sufficient growth can be reduced by introducing grid compression as discussed in sub-section 4.3.1

\section{Conclusions}

In this paper we derive a PML for the second order formulation of linear, anisotropic elastodynamics in two space dimensions. The layer equations are derived by applying a complex coordinate stretching directly to the second order equations. The resulting system is strongly hyperbolic. By a standard perturbation argument our PML at constant coefficients suffers from the same high frequency instability as the modal PML and the split field PML if the geometric stability condition is violated, while the complex frequency shift has a stabilization effect. However, in computations using standard second order finite differences our PML behaves much better than a standard first order PML. We have found several reasons for this.

In a discrete setting the unstable modes may be of higher frequency than can be represented, or well represented, on the grid. The temporal behaviour of such modes cannot be expected to be predicted by continous analysis. In fact, a straight forward computations of the temporal eigenvalues corresponding to our discrete spatial operator in a constant coefficient setting shows that if unstable modes are not well resolved they are in fact stable. Computations using different resolutions verifies the conclusion also for the variable coefficient setting. We also show that this effect can be enhanced by coordinate compression in the layer. However, coordinate compression increases the stiffness of the problem. 
Secondly, we observe that the geometric instability gives rise to growing modes, with bulk localized to part of the layer, and propagating tangentially. If a Cartesian domain is surrounded by layers, the bulk of the unstable mode eventually moves into a corner region, and decays. We analyze the stability properties of the corner region as before, finding that there is no high frequency instability. We have also observed that even if the geometric stability condition is severely violated, on a reasonably fine mesh the discrete PML is stable.

Due to these types of behaviour we have been able to construct discretely stable layers that yield reflections below the level of interior numerical errors for a veriety of anisotropic materials. However, if the violation of the geometric stability condition is strong, and a very low error level is required, the computational work required may increase dramatically.

\section{References}

[1] B. Sjogreen, N. A. Petersson. Perfectly Matched Layer for Maxwell's Equations in Second Order Formulation. Journal of Computational Physics 209 (2005) 19-46

[2] H.-O. Kreiss, N.A. Petersson, J. Ystrom. Difference Approximations for Second Order Wave Equation, SIAM Journal of Numerical Analysis 40 (2002) 1940-459.

[3] J. P. Berenger. A perfectly Matched Layer for the Absorption of Electromagnetic Waves Journal of Computational Physics 114 (1994) 185-200.

[4] E. Becache, S. Fauqueux, P. Joly. Stability of Perfectly Matched Layers, Group Velocities and Anisotropic Waves. Journal of Computational Physics 188 (2003) 399-433.

[5] D. Appelo and G. Kreiss, A New Absorbing Layer for Elastic Waves. Journal of Computational Physics 215 (2006) 642-660.

[6] A. Taflove. Advances in Computational Electrodynamics, The Finite-Difference TimeDomain. Artec House Inc. 1998

[7] J. Diaz and P. Joly Stabilized Perfectly Matched Layer for Advective Acoustics Waves 2003115 119

[8] E. A. Skelton, S. D. M. Adams, R. V. Craster. Guided Elastic Waves and Perfectly Matched Layers. Wave Motion 44 (2007) 573-592.

[9] S. Abarbanel, D. Gottlieb and J. S. Hesthaven. Long Time Behaviour of the Perfectly Matched Layer Equations in Computational Electromagnetics. Journal of Scientific Computing Vol. 17 , Nos 1-4 2002

[10] D. Appelo, A. N. Petersson. A Stable Finite Difference Method for the Elastic Wave Equation On Complex Domains With Free Surfaces. Communications in Computational Physics, Vol. 5, 84-107, 2009.

[11] S. Abarbanel and D. Gottlieb. A Mathematical Analysis of the PML Method Journal of Computational Physics 134 357-363 (1997)

[12] B. Gustafsson, H-O. Kreiss, J. Oliger. Time Dependent Problems and Difference Methods John Wileys and Sons 1995

[13] S. Nilsson, N.A. Petersson, B. Sjogreen, H.-O. Kreiss. Stable Difference Approximations for the Elastic Wave Equation in Second Order Formulation, SIAM Journal of Numerical Analysis 42 (2004) 1292-1323. 
[14] M. Kuzuoglu and R. Mittra. Frequency Dependence of the Constitutive Parameters of Causal Perfectly Matched Anisotropic Absorbers. IEEE Microwave and Guided Wave Letters Vol 6 No 121996

[15] J. A. Roden and D. S. Gedney. Convolutional PML (CPML): An Efficient FDTD Implementation of the CFS-PML for Arbitrary Media. Microwave and Optical Technology Letters 27 no. 5 334-339.

[16] K. C. Meza-Fajardo and A. S. Papageogiou. A Nonconvolutional, Split-Field, Perfectly Matched Layer for Wave Propagation in Isotropic and Anisotropic Elastic Media: Stability Analysis. Bulletin of the Seismological Society of America. Vol. 98, No. 4, 1811-1836 (2008)

[17] W. Chew and W. Weedon. A 3-D Perfectly Matched Medium from Modified Maxwell's Equations with Stretched Coordinates. Micro. Opt. Tech. Lett., Vol. 7, No. 13 599-604 (1994)

[18] D. Komatitsch and J. Tromp. A Perfectly Matched Layer Absorbing Boundary Condition for the Second Order seismic Wave Equation. Geophys. Journal International (2003) 154 146-153.

[19] E. Becache, P. G. Petropoulos, and S. D. Gedney. On the Long-Time Behaviour of Unplit Perfectly Matched Layers IEEE Transactions on Antennas and Propagation, Vol. 52 N0. 5 2004

[20] P. Gallina. Effect of Damping on Assymetric Systems. Journal of Vibration and Acoustics, Vol. 1252003.

[21] D. Appelo, T. Colonius. A high-order super-grid absorbing layer and its application to linear hyperbolic systems

[22] L. Thomsen. Weak Elastic Anisotropy. Geophysics Vol. 51 No 10 1954-1966 (1986).

[23] F. Collino. and C. Tsogka., Application of the PML absorbing layer model to the linear elastodynamic problem in anisotropic heterogeneous media, Geophysics 66, 294-307 (2001) 